\title{
Nodal sampling: a new image reconstruction algorithm for SMOS
}

\author{
Verónica González-Gambau, Antonio Turiel, Member, IEEE, Estrella Olmedo, Justino Martínez, \\ Ignasi Corbella, Adriano Camps, Fellow, IEEE,
}

\begin{abstract}
SMOS brightness temperature images and calibrated visibilities are related by the so-called G-matrix. Due to the incomplete sampling at some spatial frequencies, sharp transitions in the brightness temperature scenes generate a Gibbs-like contamination ringing and spread sidelobes. In the current SMOS image reconstruction strategy, a Blackman window is applied to the Fourier components of the brightness temperatures to diminish the amplitude of artifacts such as ripples, and other Gibbs-like effects. In this work, a novel image reconstruction algorithm focused on the reduction of Gibbs-like contamination in brightness temperature images is proposed. It is based on sampling the brightness temperature images at the nodal points, that is, at those points at which the oscillating interference causes the minimum distortion to the geophysical signal. Results show a significant reduction of ripples and sidelobes in strongly RFI-contaminated images. This technique has been throughly validated using snapshots over the ocean, by comparing brightness temperatures reconstructed in the standard way or using the nodal sampling with modeled brightness temperatures. Tests have revealed that the standard deviation of the difference between the measurement and the model is reduced around $1 \mathrm{~K}$ over clean and stable zones when using nodal sampling technique with respect to the SMOS image reconstruction baseline. The reduction is approximately $0.7 \mathrm{~K}$ when considering the global ocean. This represents a crucial improvement in brightness temperature quality, which will translate in an enhancement of the retrieved geophysical parameters, especially the sea surface salinity.
\end{abstract}

\section{Index Terms}

SMOS, interferometric radiometer, image reconstruction, nodal sampling, nodal points, sidelobes, Radio Frequency Interferences

\section{INTRODUCTION}

The European Space Agency Soil Moisture and Ocean Salinity (SMOS) is the first satellite mission devoted to the remote observation of soil moisture over land and of surface salinity over the oceans [1]-[3]. SMOS single payload is the Microwave Imaging Radiometer by Aperture Synthesis (MIRAS), being the first L-band 2D synthetic

V. González-Gambau, A. Turiel, E. Olmedo and J. Martínez are with the Department of Physical Oceanography, Institute of Marine Sciences, CSIC and with Barcelona Expert Center, Passeig Maritim de la Barceloneta, 37-49, 08003 Barcelona, Spain

I. Corbella and A. Camps are with the Remote Sensing Laboratory, Signal Theory and Communications Department, Universitat Politècnica de Catalunya, 08034 Barcelona, Spain 
aperture radiometer in orbit. MIRAS consists of 69 antennas disposed on a Y-shaped configuration with multiangular observation and full polarimetric capabilities [4], [5].

Its principle of operation is based on measuring the complex cross-correlation of the signals collected by each pair of receiving elements, providing the samples of the so-called visibility function [6]. Visibility samples are corrected from instrumental errors and denormalized by means of the calibration procedures (throughly detailed in [7]), before applying an image reconstruction algorithm to obtain brightness temperatures [8]. Visibilities are measured at selected points of the spatial frequency domain, which for the specific Y-shape geometry of MIRAS correspond to a star-shaped subarray (Fig. 1, red part) of a hexagonal grid. The G-matrix transforms the time domain brightness temperatures into visibility samples defined in the spatial frequency domain [9]. To retrieve the brightness temperatures univocally from the visibility samples, these must be defined on the full hexagon (Fig. 1, envelope of blue part). Hence, the application of the G-matrix requires to introduce zero coefficients at high spatial frequencies (blue triangles in Fig. 1). These missing Fourier coefficients generate Gibbs-like contamination and spread sidelobes from sharp transitions in the brightness temperature scenes. This is the case of images with presence of Radio-Frequency Interference (RFI) sources, affected by Sun contamination, or even images presenting land/sea/ice transitions.

SMOS operates at L-band, a protected frequency band for radio-astronomy and passive microwave remote sensing according to international regulations. However, RFI has been detected since the first measurements in-orbit during the SMOS Commissioning Phase, particularly over Europe, Middle East, and Southern and Eastern Asia [10]. These RFI sources degrade the accuracy of the retrieval of soil moisture in the areas where they are located, and also hamper the salinity retrievals in zones such as the Mediterranean Sea, China Sea and North Atlantic Ocean. Owing to intense efforts by ESA many RFI sources have been switched off, but there are still many others which make the retrieval of ocean salinity in some coastal areas impossible, and also strongly affect soil moisture retrievals. The SMOS community is making important efforts to improve RFI detection and geolocation algorithms and to develop methods for their mitigation [11]-[15]. Besides, direct Sun contamination entering as an alias in the extended aliasfree field of view also causes strong tails and ripples [16]. This is specially dramatic in the case of the ocean, as the sensitivity of L-band brightness temperatures to ocean salinity is quite low, so any perturbation of a few Kelvin causes large deviations on the retrieved salinities [17]. Much work has been directed to both mitigation and flagging of RFI [18] and Sun influence [19] on ocean scenes.

In this context, a novel brightness temperature reconstruction algorithm, focused on the improvement of Gibbs-like contamination, is presented in this study.

\section{Classical image Reconstruction SCHEME}

In an ideal case, i.e. if all the antenna patterns were equal and the decorrelation effects were considered negligible, brightness temperatures could be directly retrieved from the calibrated visibilities by applying an inverse Fourier Transform [20]. In the SMOS case, antenna patterns present non-negligible differences. To correct for these differences, a more general linear transformation, called G-matrix, is required to retrieve brightness temperatures [9]. 
A column of the G-matrix can be seen as the instrument impulse response to a point source located at a particular direction. Efficient inversion methods require the use of a hexagonal reciprocal grid for the spatial frequency coverage [20].

The current reconstruction scheme is based on zero-padding the missing spatial frequencies in the fundamental hexagon, as shown in Fig. 1 and then applying the inverse of the G-matrix. This is equivalent to assume that the visibilities at the zero-padded frequencies are negligible. If the image is smooth except at a few given places where it experiences a moderate jump in value (mathematically this is finite-variation signal), the amplitude of Fourier coefficients must decay at least as fast as the inverse of the wavenumber [21]. Henceforth, Fourier coefficients of a finite-variation signal become very small for large wavenumbers, and hence it is safe to neglect the visibilities at higher spatial frequencies if the spatial resolution is large enough (i.e., the largest wavenumbers attain high values). However, MIRAS images have not very fine resolution, and at some instances there are too many high-amplitude jumps (e.g., RFI sources). For that reason, the incomplete sampling of visibilities at higher frequencies causes some ripples that are quite evident in SMOS brightness temperature snapshots.

This problem has been circumvented in the SMOS operational processor by the application of a Blackman window on the Fourier components of the brightness temperatures. This windowing quite effectively reduces the amplitude of such ripples and improve sensitivity, with a small loss in effective spatial resolution [22], [23]. However, even after applying a Blackman window, the tails originated by large, rather punctual sources are still very evident. This effect can be clearly appreciated in the brightness temperature (TB) image of Fig. 2, corresponding to a TB image over the Pacific Ocean strongly contaminated by Sun aliases and their tails. Current SMOS image reconstruction approach (G-matrix + Blackman window) is referred in this study as the nominal reconstruction.

\section{ChANGing ThE RECONSTRUCTION PARADIGM: SPATIAL OVERSAMPLING AND NODAL POINTS}

Any sharp transition in the brightness temperature (such as RFI sources, the Sun contamination or even land/sea/ice transitions) produces a Gibbs-like effect (ringing). Therefore, in the most general case, the signal to be retrieved is a mixture of the geophysical signal (continuous signal) and the perturbation induced by some sources, as shown in the 1D representation (Fig. 3).

A new reconstruction approach is proposed based on sampling the signal at those points where eventual punctualsource perturbations, as seen by the imperfect instrument, cancel without strongly altering the value at those locations where the signal is not perturbed. By increasing the spatial sampling of the signal, the oscillating structures become clearer and those points where contamination vanishes can be estimated. The points at which the perturbations cancel are called nodal points (marked as black squares in Fig. 3). For a given signal, a subsampling of the oversampled image (with the same dimensions as in the nominal image) will be defined which verifies that the impact by distorsions (tails, ripples) is minimal. This selection of points of the oversampled image is called Nodal Sampling (NS).

The underlying hypothesis of the NS is that the geophysical signal of interest, at the scale of the spatial resolution of the instrument, varies relatively slowly except at the edges of the different regions forming the image. Therefore, 
it is not expected to have large gradients all over the image. This is very convenient to remove tails and ripples originated by RFI sources and Sun, but also by coastlines. At those points where there is no perturbation, the subsampled signal will take a value which is the result of the interpolation among the points nearby, which introduces some error. However, if the signal changes slowly this error will be rather small. This approximation is very pertinent specially in the case of the sea, as ocean structures have typical scales of tens to hundreds of kilometers.

\section{A. Spatial oversampling}

The spatial oversampling of the brigthness temperature image is performed by embedding the Fourier coefficients of the fundamental hexagon in a larger hexagon, with the new spatial frequencies zero-padded. This process grants that the original information is completely preserved during the oversampling process, as the original Fourier coefficients are kept.

All the Fourier Transforms in this study have been performed using the change of coordinates defined in [20] in order to apply the standard rectangular FFT routines to hexagonally sampled signals, so all the domains are squared. The original domain is of side $l=64$, hence containing $64 \times 64=4096$ Fourier coefficients. The matrix of Fourier coefficients is embedded in a larger square domain of side $L$, with an integer relation $\beta$ between $l$ and $L: L=\beta l$ (for computational reasons it is very convenient to take $\beta$ an odd number). All new Fourier coefficients are set to zero, while the other are multiplied by $\beta^{2}$ to ensure proper normalization. An inverse Fourier Transform is applied to the larger Fourier domain in order to obtain an oversampled brightness temperature image, denoted by $T$, which has the same spatial coverage as the original image $t$, but is $\beta^{2}$ times denser.

By construction, oversampled images depend continuously on the parameter $\beta$ (what in turn means that any image can be extrapolated in a consistent way to any desired resolution), as we prove in the following. Let $\left\{t\left(x_{m}, y_{n}\right)\right\}_{m, n=0, \ldots, N-1}$ be a uniformly sampled signal with NxN samples, with sampling points defined by the coordinates $x_{m}=x_{0}+m \delta x, y_{n}=y_{0}+n \delta y$. To simplify notation, from now on $t\left(x_{m}, y_{n}\right) \equiv t(m, n)$. Its Fourier transform is hence

$$
\hat{t}(k, l)=\sum_{l=0}^{N-1} \sum_{k=0}^{N-1} t(m, n) e^{-2 \pi i\left(\frac{k m+l n}{N}\right)}
$$

where $\hat{t}(m, n)$ stands for the Fourier coefficient of the brightness temperature image at the original resolution grid with normalized coordinates $(m, n)$ in Fourier space.

According to our construction, the Fourier coefficients of the oversampled image $\hat{T}(\delta, \gamma)$ are given by the following expression: 


$$
\hat{T}(\delta, \gamma)=\left\{\begin{array}{lll}
\beta^{2} \hat{t}(\delta, \gamma) & 0 \leq \delta<N / 2 & ; 0 \leq \gamma<N / 2 \\
\beta^{2} \hat{t}\left(\delta-N_{L}+N, \gamma\right) & N_{L}-N / 2 \leq \delta \leq N_{L}-1 & ; 0 \leq \gamma<N / 2 \\
\beta^{2} \hat{t}\left(\delta, \gamma-N_{L}+N\right) & 0 \leq \delta<N / 2 & ; \quad N_{L}-N / 2 \leq \gamma \leq N_{L}-1 \\
\beta^{2} \hat{t}\left(\delta-N_{L}+N, \gamma-N_{L}+N\right) & N_{L}-N / 2 \leq \delta \leq N_{L}-1 & ; \quad N_{L}-N / 2 \leq \gamma \leq N_{L}-1 \\
0 & \text { otherwise } &
\end{array}\right.
$$

where $N_{L}=\beta N$. Notice that we have multiplied the Fourier coefficients of the original image by $\beta^{2}$ to ensure proper normalization, that is, that the amplitude of the oversampled signal in direct space is the same as the amplitude of the original signal. The oversampled image, denoted by $T\left(X_{\mu}, Y_{\nu}\right)$ or shortly by $T(\mu, \nu)$ to simplify notation, is given by:

$$
\begin{aligned}
T(\mu, \nu)= & \frac{1}{N_{L}^{2}} \sum_{\gamma=0}^{N_{L}-1} \sum_{\delta=0}^{N_{L}-1} \hat{T}(\delta, \gamma) e^{2 \pi i\left(\frac{\mu \delta+\nu \gamma}{N_{L}}\right)}= \\
= & \frac{1}{N^{2}} \sum_{\gamma=0}^{N / 2-1} \sum_{\delta=0}^{N-1} \hat{t}(\delta, \gamma) e^{2 \pi i\left(\frac{\mu \delta+\nu \gamma}{N_{L}}\right)}+\frac{1}{N^{2}} \sum_{\gamma=0}^{N / 2-1} \sum_{\delta=N / 2}^{N-1} \hat{t}(\delta, \gamma) e^{2 \pi i\left(\frac{\mu(\delta-N)+\nu \gamma}{N_{L}}\right)}+ \\
& +\frac{1}{N^{2}} \sum_{\gamma=N / 2}^{N-1} \sum_{\delta=N / 2}^{N-1} \hat{t}(\delta, \gamma) e^{2 \pi i\left(\frac{\mu(\delta-N)+\nu(\gamma-N)}{N_{L}}\right)}+\frac{1}{N^{2}} \sum_{\gamma=N / 2}^{N-1} \sum_{\delta=0}^{N / 2-1} \hat{t}(\delta, \gamma) e^{2 \pi i\left(\frac{\mu \delta+\nu(\gamma-N)}{N_{L}}\right)}
\end{aligned}
$$

The sampling points in the denser grid $\left(X_{\mu}, Y_{\nu}\right)$ are given by $X_{\mu}=x_{0}+\mu \frac{\delta x}{\beta}, Y_{\nu}=y_{0}+\nu \frac{\delta y}{\beta}$, where $\mu, \nu=$ $0, \ldots, N_{L}-1$. Note that in the second and third summations the dummy summation index $\delta$ has been redefined, changing it by $\delta-N_{L}+N$ and in the third and fourth summations the dummy summation index $\gamma$ has been redefined, changing it by $\gamma-N_{L}+N$.

Interestingly, if $\mu=\beta m$ and $\nu=\beta n$, for integers values of $m$ and $n$, we have $\left(X_{\mu}, Y_{\nu}\right)=\left(x_{m}, y_{n}\right)$ and

$$
T(m, n)=\frac{1}{N^{2}} \sum_{\gamma=0}^{N-1} \sum_{\delta=0}^{N-1} \hat{t}(\delta, \gamma) e^{2 \pi i\left(\frac{m \delta+n \gamma}{N}\right)}=t(m, n)
$$

so the oversampled image takes exactly the same values as the original ones in the original sampling points. Note also that taking $\Delta x=N \delta x, \Delta y=N \delta y$, defining $\alpha=\frac{\mu}{N_{L}}$ and $\alpha^{\prime}=\frac{\nu}{N_{L}}$, and substituting them in eq. (3), it is obtained that:

$$
\begin{array}{r}
T\left(x_{0}+\alpha \Delta x, y_{0}+\alpha^{\prime} \Delta y\right)=\frac{1}{N^{2}} \sum_{\gamma=0}^{N / 2-1} \sum_{\delta=0}^{N-1} \hat{t}(\delta, \gamma) e^{2 \pi i\left(\alpha \delta+\alpha^{\prime} \gamma\right)}+\frac{1}{N^{2}} \sum_{\gamma=0}^{N / 2-1} \sum_{\delta=N / 2}^{N-1} \hat{t}(\delta, \gamma) e^{2 \pi i\left(\alpha(\delta-N)+\alpha^{\prime} \gamma\right)} \\
+\frac{1}{N^{2}} \sum_{\gamma=N / 2}^{N-1} \sum_{\delta=N / 2}^{N-1} \hat{t}(\delta, \gamma) e^{2 \pi i\left(\alpha(\delta-N)+\alpha^{\prime}(\gamma-N)\right)}+\frac{1}{N^{2}} \sum_{\gamma=N / 2}^{N-1} \sum_{\delta=0}^{N / 2-1} \hat{t}(\delta, \gamma) e^{2 \pi i\left(\alpha \delta+\alpha^{\prime}(\gamma-N)\right)}
\end{array}
$$

which is a continuous function of $\left(\alpha, \alpha^{\prime}\right)$ as it is a finite sum of continuous functions of $\left(\alpha, \alpha^{\prime}\right)$. Thus, the oversampled image takes the same values as the original image at those sampling points $\left(X_{\mu}, Y_{\nu}\right)=\left(x_{m}, y_{n}\right)$, and 
it depends continuously on the oversampling variables $\alpha$ and $\alpha^{\prime}$. We can now try to locate those points at which the oscillatory perturbations by tails or ripples cancel, so the values on those points will be exactly those of the geophysical signal. For a given oversampling factor $\beta, \alpha$ and $\alpha^{\prime}$ take discrete values $\alpha=\frac{\mu}{\beta N}$ and $\alpha^{\prime}=\frac{\nu}{\beta N}$, where $\mu, \nu=0, \ldots, \beta N-1$. Therefore, with a proper value of $\beta$ it is possible to oversample the image with enough accuracy to approach the ideal nodal sampling.

An example of the original $(t)$ and the oversampled $(T)$ brightness temperature images is presented in Figure 4. An oversampling factor $\beta=9$ has been used. As it can be seen in this figure, the tails spawning from the RFI source at the original image (Fig. 4a) have a clearly oscillating aspect in the oversampled image (Fig. 4b), what means that the perturbation must cancel in the space between a peak and a valley.

\section{B. Method to obtain the Nodal Sampling}

Finding the NS is not simple, as the value of the geophysical signal is not known, neither a model of the perturbation is available. However, nodal points can be characterized by some of their functional properties. Let us first pose the problem in one dimension. The points at which the perturbations cancel, the nodal points, are also the points at which the gradient takes a local maximum value: nodal points are inflection points, at which the negative curvature of a peak changes to a positive curvature of the valley, and hence the rate of variation of the signal is maximum. As the geophysical signal, by hypothesis, has much smaller gradients almost everywhere, the gradient of the signal will be dominated by the gradient of the perturbation when it is present, and especially at nodal points. Hence, as a first approximation the nodal sampling will be given by the local maxima of the gradient, that is, by the zeroes of the second derivative of the oversampled image. In two dimensions the situation is slightly more complicated. The NS will be given by the local minima of the Laplacian of the oversampled image, as the second derivative along the oscillating direction cancels, and it is small (almost flat) along the orthogonal direction. Interestingly, for a given percusion on a elastic membrane (e.g., a drum) the points that do not oscillate are called nodal points and they are determined by the condition of vanishing Laplacian.

In the lines below we will provide a step-by-step description of the algorithm used to estimate the approximation to nodal sampling when a parameter $\beta$ is given, but let us first provide a simplified overview of it. In the first step of the algorithm, the original image with $N \times N$ samples is oversampled. The oversampled image is a new image with $N_{L} \times N_{L}$ samples $\left(N_{L}=\beta N\right)$, so that each point in the original image is associated to $\beta^{2}$ points of the oversampled grid, which correspond to the subpixels included in the area covered by the original pixel. The following steps of the algorithm aim to obtain a corrected image with the same number of samples as the original one, that is, $N \times N$. This corrected image is constructed by taking the values of the oversampled image at specific points, each point in the corrected image being one specific point of its associated $\beta^{2}$ subpoints. The choice of points in the oversampled grid leading to the corrected image is iteratively refined, but the oversampled image is never changed, just the grid. By construction, the points in the corrected image are assigned to the same physical positions of those of the original image. The displacement implied by taking a subpixel different from the one at the center of the pixel is smaller than the pixel size, so we accept this representativity error as it is expected 
to be smaller than the error caused by Gibbs effects. The grid of selected points of the oversampled image is modified until attaining a quality goal, which implies that the impact of the contaminations in the corrected image is minimum. At that moment, we have the approximation to the nodal sampling grid for the given oversampling factor $\beta$.

The algorithm proceeds as follows:

1) The oversampled brightness temperature image $T(\mu, \nu)$ is obtained as described in section III-A with a given oversampling factor $\beta$ and oversampling points $\left\{X_{\mu}, Y_{\nu}\right\}_{\mu, \nu=0, \ldots, \beta N-1}$.

2) The high-resolution Laplacian of the oversampled image, $\Delta_{H} T(\mu, \nu)$, is computed at each point as the difference between the average of the TB values at the six first neighbours of that point (according to SMOS hexagonal geometry, see [20]) and the value at that point [24]; namely:

$$
\begin{aligned}
\Delta_{H} T(\mu, \nu) \equiv & {[T(\mu+1, \nu)+T(\mu-1, \nu)+T(\mu, \nu+1)+} \\
& T(\mu, \nu-1)+T(\mu+1, \nu-1)+T(\mu-1, \nu+1)] / 6-T(\mu, \nu)
\end{aligned}
$$

3) The first approximation to the set of nodal points in the oversampled image, denoted by $\left\{X_{\mu_{0}(m, n)}, Y_{\nu_{0}(m, n)}\right\}_{m, n=0, \ldots, N-1}$, is calculated as the local minima of the high-resolution Laplacian at each $\beta \times \beta$ block (corresponding to each pixel in the original image), i.e., $m \beta \leq \mu_{0}(m, n)<(m+1) \beta$, $n \beta \leq \nu_{0}(m, n)<(n+1) \beta$, and

$$
\begin{array}{r}
\left|\Delta_{H} T\left(\mu_{0}(m, n), \nu_{0}(m, n)\right)\right| \leq\left|\Delta_{H} T(\mu(m, n), \nu(m, n))\right| \\
\forall \mu, \nu: m \beta \leq \mu(m, n)<(m+1) \beta, n \beta \leq \nu(m, n)<(n+1) \beta
\end{array}
$$

4) Start of the iterative loop: given the current $i$-th approximation to the nodal grid, $\left\{X_{\mu_{i}(m, n)}, Y_{\nu_{i}(m, n)}\right\}$, the corrected image $t_{i}$ at the original resolution, defined by $t_{i}(m, n) \equiv T\left(\mu_{i}(m, n), \nu_{i}(m, n)\right)$, is obtained.

5) The current selection of nodal points is iteratively fine tuned: For each position in the coarse grid $(m, n)$, at the i-th iteration:

5.1 The average value of the six first neighbours of the current corrected image is computed. It is denoted by $\bar{t}_{i}(m, n)$, and it is given by:

$$
\begin{aligned}
\bar{t}_{i}(m, n) \equiv & \left(t_{i}(m+1, n)+t_{i}(m-1, n)+t_{i}(m, n+1)+\right. \\
& \left.t_{i}(m, n-1)+t_{i}(m+1, n-1)+t_{i}(m-1, n+1)\right) / 6
\end{aligned}
$$

5.2 A new oversampling point $\left\{X_{\mu_{i+1}(m, n)}, Y_{\nu_{i+1}(m, n)}\right\}$ is searched for, verifying that the lower resolution Laplacian, $\Delta_{L} t_{i+1}(m, n)$ would be reduced in absolute value if the corrected image at that point were substituted with the new oversampling point; more precisely, defining the lower-resolution Laplacian estimate as 


$$
\hat{\Delta}_{L_{i+1}} T(m, n) \equiv \bar{t}_{i}(m, n)-T\left(\mu_{i+1}(m, n), \nu_{i+1}(m, n)\right)
$$

where $\left\{X_{\mu_{i+1}(m, n)}, Y_{\nu_{i+1}(m, n)}\right\}$ is defined such that $m \beta \leq \mu_{i+1}(m, n)<(m+1) \beta, n \beta \leq \nu_{i+1}(m, n)<$ $(n+1) \beta$ and

$$
\begin{array}{r}
\left|\hat{\Delta}_{L_{i+1}} T(m, n)\right| \leq\left|\bar{t}_{i}(m, n)-T(\mu, \nu)\right| \\
\forall \nu, \mu: m \beta \leq \mu<(m+1) \beta, n \beta \leq \nu<(n+1) \beta
\end{array}
$$

5.3 End of iterative loop: The algorithm is iterated from 4) until a break condition is reached (the reason for a break condition being explained later).

In Fig. 5 three different subgrids on an oversampled image are presented; the background brightness temperature image is a zoom of the oversampled image in Fig. 4b. White stars indicate which points belong to each subgrid. Fig. 5a presents the regular $N \times N$ subgrid which leads to the same values of brightness tempeatures as the original image. The initial guess of nodal points (step 3) is shown in Fig. 5b. The brightness temperature image reconstructed sampling the signal at that subgrid is shown in Fig. 6b. Nodal points estimated after 20 iterations are depicted in Fig. 5c. From these images it is clear how the nodal points selection has been fine tuned after 20 iterations and that nodal points have concentrated in the spaces between a peak and a valley. Final reconstructed brightness temperature is obtained sampling the original image at the final selection of nodal points (Fig. 6c). A significant reduction of the general ripples and tails in this image can be appreciated when comparing it to the nominal reconstruction (Fig. 6a).

The break condition in 6.3 can be defined in several ways, typically associated to the increase in quality of the signal. As it will be discussed in the results section, the spatial standard deviation of the corrected images rapidly decreases (indicating that spurious large amplitude oscillations are being removed, as confirmed by visual inspection) during the first iterations and then stagnates. The number of points being updated also decreases very rapidly until it attains a non-zero minimum.

Note that, as many points may be updated when passing from the i-th iteration to the (i+1)-th iteration, the lower-resolution Laplacian estimate, $\hat{\Delta}_{L_{i+1}} T(m, n)$, needs not to coincide with the lower-resolution Laplacian of the (i+1)-th corrected image, $\Delta_{L} t_{i+1}(m, n)=\bar{t}_{i+1}(m, n)-t_{i+1}(m, n)$. This may eventually lead to an oscillatory behaviour, in which the same points get updated to one value then back to the previous one. This seems to happen if no break condition is introduced. For the sake of simplicity, in the present version of the algorithm a very simple break condition has been introduced: the algorithm stops when 20 iterations have been reached, as from all the tests performed no significant image improvement has been observed after this amount of iterations.

The nodal sampling technique has been implemented using as input data the Fourier components of the brightness temperatures generated by MIRAS Testing Software (MTS) [25]. Nodal sampling has been applied to the images presented in this section using an oversampling factor $\beta=9$ and the number of iterations has been fixed to 20 . 
The computational time of running the nodal sampling algorithm under these conditions is around 4 minutes per orbit using a standard workstation. This compares favourably the computational time required for the standard processing from level 0 (raw data) to level 1B (brightness temperatures) using the MTS software, which takes around 20 minutes. The nodal sampling algorithm could be straightforwardly extended to a domain of side $1=128$, as the one used in the SMOS Level 1 Operational Processor.

\section{Results}

As discussed in the sections above, the reduction of sidelobes is particularly crucial for sea surface salinity, where very small variations of brightness temperatures are important for a quality retrieval. In this paper, the algorithm performance has been throughly analyzed using snapshots over the ocean. For all the images presented in this manuscript, brightness temperatures are at the antenna reference frame. Overlaying the TB images, the red line gives the fundamental hexagon in the $(\xi, \eta)$ antenna reference frame and the blue line of the inner curved hexagon limited by the unity circle contours (dashed lines) defines the Nyquist Alias Free-Field of View (AF-FOV) [26].

An example of an RFI source produced by a ship on the Atlantic Ocean has been used to illustrate the nodal sampling performance in strongly RFI-contaminated images. A point source (such as an RFI source or the Sun) is seen by the instrument not only as a single strong point source, but also as a systematic structure with visible sidelobes (so-called tails) at $\pm 30, \pm 90$ and \pm 150 degrees with respect to the positive $\mathrm{x}$-axis from the RFI source centre, that contaminate other parts of the image. This can be clearly appreciated in the brightness temperatures corresponding to the current image reconstruction approach in Fig. 7a. The associated brightness temperature image in the fundamental hexagon after applying the nodal sampling algorithm is shown in Fig. 7b. Comparing this image to the nominal one, a clear reduction of the general ripples and the sidelobes along the RFI directions can be appreciated. As shown in the histograms of TB values, the distribution of brightness temperatures has been concentrated in the expected range for the geophysical signal when NS is applied (Fig. 7d) with respect to the nominal processing (Fig. 7c). Red bars in the histograms account for negative values and those values higher than $350 \mathrm{~K}$ (i.e. non-natural emission). As evidenced by the graphs, the bulk of the negative brightness temperatures has been removed by sampling the signal at the selected nodal points. A part of the negative TB values in the nominal image have become values higher than $350 \mathrm{~K}$ in the nodal sampled brightness temperature image. This fact explains the increase of the excess bar accounting for those values higher than $350 \mathrm{~K}$. However, considering a mask of the brightness temperature images (pixels corresponding to non-natural emission, that is negative TB and values higher than $350 \mathrm{~K}$, have been marked) it can be observed that points assigned by the NS (Fig. 7f) to values higher than $350 \mathrm{~K}$ correspond to values which are also contaminated in the nominal image (Fig. 7e). In this image, the RFI source has a wider extent after applying nodal sampling than in the nominal case. This widening of sources seems to happen with very deteriorated signals and, according to our experiences is not a systematic low-pass filter effect but rather a non-linear adaptive interpolation. This can be clearly seen in Fig. 6, where some RFI sources present this widening effect with respect to the TB image in the nominal case (Fig. 6a), but not some others, such as the ones located around $(\xi, \eta)$ positions $(0.28,0.04)$ and $(0.25,0.41)$, which in fact have been removed using NS (Fig. 
6c). Cuts of the brightness temperature image along the directions of the RFI show a quite significant reduction of the sidelobe levels (Fig. 8; note that the scale of the y-axis is logarithmic to better show the full variability of brightness temperatures). It can be clearly observed that using the proposed approach (blue line), sidelobes levels have been considerably decreased and the TB is smoother than in the nominal processing (black line). These results have been compared with those from a clean snapshot taken 18 days later (as the satellite overpassed the same zone), when no RFI was present (Fig. 9). Cuts of the clean image, performed along the directions shown in Fig. 8d, are presented in Fig. 10. Results indicate that nodal sampling is reducing the small ripples present in the image even in the clean scene while maintaining the geophysical contributions.

To further assess if the geophysical signal is preserved when nodal sampling is applied, SMOS TB measurements over ocean have been compared with modeled brightness temperatures, derived from the Geophysical Model Function (GMF) presented in [27], and applying it to geophysical priors [28]. Then, the difference between both brightness temperatures has been computed and the statistics of this new variable have been evaluated. In this analysis, an ascending overpass in a very stable zone over the Pacific Ocean (longitude-latitude coordinates: [137 $\mathrm{W}-95^{\circ} \mathrm{W}, 45^{\circ} \mathrm{S}-5^{\circ} \mathrm{S}$ ], where the operational OTT -Ocean Target Transformation- is computed [29], [30], has been used. The OTT is used in the operational processing to correct for residual antenna-frame systematic errors. The median for all the snapshots over that zone of the brightness temperatures difference per each point in the Extended Alias-Free Field of View (EAF-FOV) region is presented in Fig. 11. Left column plots correspond to the nominal and right column plots to the nodal sampled images. Results correspond to X (top row) and Y-polarization (bottom row), using only pure dual epochs, that is, epochs with all arms at the same polarization status, either $\mathrm{X}$ or $\mathrm{Y}$ [31]. The statistics computed for all the points in the AF-FOV and EAF-FOV regions are also indicated in the plots. As shown in the figure, spatial structures observed in the brightness temperatures difference derived with the nominal processing can also be recognised in NS case, although the later are smoother, as indicated by the standard deviation. This means that NS is correcting rapidly-oscillating errors but without introducing changes in the behaviour of the systematic antenna-frame errors.

To characterize the extent of the error reduction when NS is applied, the standard deviation for all the snapshots over that zone of the difference between measured and modeled brightness temperatures has been computed for each point in the EAF-FOV region. Figure 12 shows that it is significantly reduced in most of the pixels using nodal sampling (right column) with respect to the nominal case (left column). Note that Sun alias and its tails can be clearly distinguished in the standard deviation plots. The histograms of those standard deviations are shown in Fig. 13. These distributions reveal that the brightness temperatures retrieved using nodal sampling are closer to the modeled ones than using the current SMOS image reconstruction baseline, since the average standard deviation has been reduced by approximately $1 \mathrm{~K}$ for both polarizations.

The study has been extended in order to see the overall performance of the nodal sampling algorithm over the Earth's oceans. A 9-day (June 12-21, 2014) $0.25^{\circ}$ resolution global map of the First Stokes brightness temperatures difference between SMOS measurements and the model [27] is presented in Fig. 14 for nominal image reconstruction and Fig. 15 for the nodal sampled brightness temperatures. In both cases, the corresponding OTT has been subtracted 
to the brightness temperatures before computing the difference with the modeled TB. Brightness temperatures at BOA (Bottom of the Atmosphere) have been used and their values in the AF-FOV region have been averaged to produce these maps. It is worth noting that using nodal sampling, the residual difference between the correctedOTT brightness temperatures and the modeled brightness temperature is significantly decreased with respect to the nominal image reconstruction. Besides, the reduction in the standard deviation of the First Stokes brightness temperature differences is approximately $0.7 \mathrm{~K}$ when nodal sampling is used (Fig. 17) with respect to the nominal image reconstruction (Fig. 16).

Since each error of $0.5 \mathrm{~K}$ represents an error of approximately $1 \mathrm{psu}$, a gain of quality of about 2 psu is expected in the salinity retrievals over clean and stable zones of the ocean when nodal sampling is introduced, and of approximately 1.4 psu over the global ocean. This gain of quality is quite considerable taking into account that the SMOS mission requirement is 1 psu of accuracy at level 2.

\section{CONCLUSIONS AND FUTURE WORK}

In this paper a new algorithm for SMOS image reconstruction, the Nodal Sampling, has been introduced. NS is based on oversampling reconstructed images by an appropriate extension in Fourier space and then subselecting a grid of points with the condition of minimum distorsion by oscillatory perturbations to reconstruct the signal. The method has shown to be easy to implement, it leads to correct general ripples and significantly reduces sidelobes which are visually quite noticeable.

Validation over ocean scenes has shown that applying Nodal Sampling as SMOS image reconstruction provides a considerable error reduction on brigthness temperatures. This reduction is of approximately $1 \mathrm{~K}$ on clean orbits as the ones used to compute the Ocean Target Transformation, and of $0.7 \mathrm{~K}$ in average over the whole ocean. Therefore, an estimated quality gain of around 1.4 psu can be expected in the salinity retrievals with Nodal Sampling.

While Nodal Sampling always diminishes the impact of tails and ripples, it has an unequal performance on the area where RFI sources are located. In some cases, the affected area is larger while in other cases this area becomes muchs smaller than in the nominal case. Both effects (increase or decrease in the size of the RFI area) can take place in the same snapshot (see for instance Figure 6), what indicates that Nodal Sampling is not equivalent to apply a linear filter (which would produce the same effect all over the image). The reasons for this different behaviour of Nodal Sampling are still under investigation. They may be related to the deterioration of the signal when a large RFI is present, that make the points close to the souce impossible to retrieve. Besides, under some circumstances the nodal points seem to lie a little beyond the boundaries of the original pixel, so a modification of the standard algorithm is required (under investigation).

The presence of the effects mentioned above open the question about which is the effective resolution of SMOS images after applying Nodal Sampling. Answering this question is not straightforward, because Nodal Sampling is not linear and so the resolution needs not to be homogeneous across the image. It should be possible to produce an assessment of the average effective resolution by analyzing the spectral content of an ensemble of snapshots, something that deserves a dedicated study. In addition, it would be very convenient to devise metrics to evaluate 
the local resolution on each snapshot; this would be helpful not only on operational terms, but also to drive the improvements in the method.

Out of RFI sources the blurring of geophysical structures seems to be not quite significant, as confirmed by the better correspondence of NS brightness temperatures with modelled ones than the same comparison with nominal brightness temperatures. It is however necessary to make a more precise assessment on the structural content of images; we are presently working on a method based on the correspondence of singularity exponents [32]. Alternative methods include the analysis of the spatial correlations of the signal at level 2 (retrieved sea surface salinities).

The next step is to assess the impact of using the Nodal Sampling technique on the quality of sea surface salinity retrievals. This is not straightforward since several aspects of the salinity inversion need to be previously addressed. For example, an estimation of the radiometric accuracies is required since NS seems to lead to a decrease of the expected errors. Furthermore, the stability of the OTT needs to be evaluated to establish an appropriate computation frequency. So, obtaining sea surface salinities from nodal sampled brightness temperatures needs a dedicated experimental set up, already in progress.

Over land, NS could also be useful to improve soil moisture retrievals, specially when the presence of RFI sources hampers quality retrievals over the land-affected areas. However, spatial correlation scales are typically smaller over land than over ocean; moreover, the requirements on spatial resolution are more stringent. As NS requires spatial gradients of the signal to be retrieved considerable smaller than those of the perturbation, NS may present lower performance on land applications. NS performance evaluation tests over land are then required. Validation over land is more complicated than over ocean since geophysical models of soil moisture have also a wider variability range. This is a subject of further research.

Finally, it is important to mention that the method presented in this paper, although used for improving the quality of SMOS images, could be used in any other context in which an image is reconstructed with partial information of its Fourier coefficients. NS will be of application if the signal to be separated from the perturbation is of relative slow variation. This can be useful to improve image quality in other interferometric instruments, as well as in image reconstruction in general.

\section{ACKNOWLEDGMENT}

This work has been funded by the Spanish Ministry of Economy through the National R+D Plan by means of MIDAS-7 project AYA2012-39356-C05-03 and previous grants.

\section{REFERENCES}

[1] H. Barre, B. Duesmann, and Y. Kerr, "SMOS: The mission and the system," Geoscience and Remote Sensing, IEEE Transactions on, vol. 46, no. 3, pp. 587-593, March 2008.

[2] Y. Kerr, P. Waldteufel, J. Wigneron, S. Delwart, F. Cabot, J. Boutin, M. Escorihuela, J. Font, N. Reul, C. Gruhier, S. Juglea, M. Drinkwater, A. Hahne, M. Martin-Neira, and S. Mecklenburg, "The SMOS mission: New tool for monitoring key elements of the global water cycle," Proceedings of the IEEE, vol. 98, no. 5, pp. 666-687, May 2010. 
[3] J. Font, A. Camps, A. Borges, M. Martin-Neira, J. Boutin, N. Reul, Y. Kerr, A. Hahne, and S. Mecklenburg, "SMOS: The challenging sea surface salinity measurement from space," Proceedings of the IEEE, vol. 98, no. 5, pp. 649-665, May 2010.

[4] M. Martin-Neira and J. Goutoule, "MIRAS: a two dimensional aperture synthesis radiometer for soil moisture and ocean salinity observations," ESA Bulletin, vol. 92, pp. 95-104, 1997.

[5] K. D. McMullan, M. Brown, M. Martin-Neira, W. Rits, S. Ekholm, J. Marti, and J. Lemanczyk, "SMOS: The payload," Geoscience and Remote Sensing, IEEE Transactions on, vol. 46, no. 3, pp. 594-605, March 2008.

[6] I. Corbella, N. Duffo, M. Vall-llossera, A. Camps, and F. Torres, "The visibility function in interferometric aperture synthesis radiometry," Geoscience and Remote Sensing, IEEE Transactions on, vol. 42, no. 8, pp. 1677-1682, Aug 2004.

[7] M. Brown, F. Torres, I. Corbella, and A. Colliander, "SMOS calibration," Geoscience and Remote Sensing, IEEE Transactions on, vol. 46, no. 3, pp. 646-658, March 2008.

[8] I. Corbella, F. Torres, A. Camps, N. Duffo, and M. Vall-llossera, "Brightness-temperature retrieval methods in synthetic aperture radiometers," Geoscience and Remote Sensing, IEEE Transactions on, vol. 47, no. 1, pp. 285-294, Jan 2009.

[9] A. Tanner and C. Swift, "Calibration of a synthetic aperture radiometer," Geoscience and Remote Sensing, IEEE Transactions on, vol. 31, no. 1, pp. 257-267, Jan 1993.

[10] R. Oliva, E. Daganzo, Y. Kerr, S. Mecklenburg, S. Nieto, P. Richaume, and C. Gruhier, "SMOS Radio Frequency Interference scenario: Status and actions taken to improve the RFI environment in the 1400-1427 MHz passive band," Geoscience and Remote Sensing, IEEE Transactions on, vol. 50, no. 5, pp. 1427-1439, May 2012.

[11] A. Camps, J. Gourrion, J. M. Tarongi, M. Vall Llossera, A. Gutierrez, J. Barbosa, and R. Castro, "Radio-Frequency Interference detection and mitigation algorithms for synthetic aperture radiometers," Algorithms, vol. 4, no. 3, pp. 155-182, 2011. [Online]. Available: http://www.mdpi.com/1999-4893/4/3/155

[12] E. Daganzo-Eusebio, R. Oliva, Y. Kerr, S. Nieto, P. Richaume, and S. Mecklenburg, "SMOS radiometer in the 1400-1427 MHz passive band: Impact of the RFI environment and approach to its mitigation and cancellation," Geoscience and Remote Sensing, IEEE Transactions on, vol. 51, no. 10, pp. 4999-5007, Oct 2013.

[13] R. Castro, A. Gutiérrez, and J. Barbosa, "A first set of techniques to detect Radio Frequency Interferences and mitigate their impact on SMOS data," Geoscience and Remote Sensing, IEEE Transactions on, vol. 50, no. 5, pp. 1440-1447, May 2012.

[14] Y. Soldo, A. Khazaal, F. Cabot, P. Richaume, E. Anterrieu, and Y. Kerr, "Mitigation of RFIs for SMOS: A distributed approach," Geoscience and Remote Sensing, IEEE Transactions on, vol. 52, no. 11, pp. 7470-7479, Nov 2014.

[15] H. Park, V. González-Gambau, and A. Camps, "High angular resolution RFI localization in synthetic aperture interferometric radiometers using Direction-Of-Arrival estimation," Geoscience and Remote Sensing Letters, IEEE, vol. 12, no. 1, pp. 102-106, Jan 2015.

[16] A. Camps, M. Vall-llossera, N. Duffo, M. Zapata, I. Corbella, F. Torres, and V. Barrena, "Sun effects in 2-d aperture synthesis radiometry imaging and their cancelation," Geoscience and Remote Sensing, IEEE Transactions on, vol. 42, no. 6, pp. 1161-1167, June 2004.

[17] S. Yueh, R. West, W. Wilson, F. K.Li, E. Njoku, and Y. Rahmat-Samii, "Error sources and feasibility for microwave remote sensing of ocean surface salinity," Geoscience and Remote Sensing, IEEE Transactions on, vol. 39, no. 5, pp. 1049-1060, May 2001.

[18] L2OS team, "Tuning L2OS RFI detection,” Argans, Tech. Rep., 2013.

[19] X. Yin, J. Boutin, and P. Spurgeon, "Biases between measured and simulated SMOS brightness temperatures over ocean: Influence of sun," Selected Topics in Applied Earth Observations and Remote Sensing, IEEE Journal of, vol. 6, no. 3, pp. 1341-1350, June 2013.

[20] A. Camps, J. Bara, I. Sanahuja, and F. Torres, "The processing of hexagonally sampled signals with standard rectangular techniques: application to 2-D large aperture synthesis interferometric radiometers," Geoscience and Remote Sensing, IEEE Transactions on, vol. 35, no. 1, pp. 183-190, Jan 1997.

[21] S. Mallat, A Wavelet Tour of Signal Processing. 2nd Edition: Academic Press, 1999.

[22] J. Bará, A. Camps, F. Torres, and I. Corbella, "Angular resolution of two-dimensional hexagonally sampled interferometric radiometers," Radio Science, vol. vol. 33, no. 5, pp. 1459-1473, 1998.

[23] E. Anterrieu, P. Waldteufel, and A. Lannes, "Apodization functions for 2-D hexagonally sampled synthetic aperture imaging radiometers," Geoscience and Remote Sensing, IEEE Transactions on, vol. 40, no. 12, pp. 2531-2542, Dec 2002.

[24] W. Zakrzewski, "Laplacians on lattices," Journal of Nonlinear Mathematical Physics, vol. 12:4, pp. 530-538, 2005.

[25] I. Corbella, F. Torres, N. Duffo, V. González, A. Camps, and M. Vall-llossera, "Fast processing tool for SMOS data," in Geoscience and Remote Sensing Symposium, 2008. IGARSS 2008. IEEE International, vol. 2, July 2008, pp. II-1152-II-1155. 
[26] A. Camps, "Application of interferometric radiometry to Earth observation," Ph.D. dissertation, Universitat Politecnica de Catalunya, 1996. [Online]. Available: http://www.tdx.cat/TDX-1020104-091741

[27] S. Guimbard, J. Gourrion, M. Portabella, A. Turiel, C. Gabarró, and J. Font, "SMOS semi-empirical ocean forward model adjustment," IEEE Transactions on Geoscience and Remote Sensing, vol. 50, pp. 1676-1687, 2012.

[28] S. Zine, J. Boutin, J. Font, N. Reul, P. Waldteufel, C. Gabarró, J. Tenerelli, F. Petitcolin, J. Vergely, M. Talone, and S. Delwart, "Overview of the SMOS sea surface salinity prototype processor," Geoscience and Remote Sensing, IEEE Transactions on, vol. 46, no. 3, pp. 621-645, March 2008.

[29] I. Meirold-Mautner, C. Mugerin, J. Vergely, P. Spurgeon, F. Rouffi, and M. Meskini, "SMOS ocean salinity performance and TB bias correction," in EGU General Assembly, 2009.

[30] J. Tenerelli and N. Reul, "Analysis of L1PP calibration approach impacts in SMOS TB and 3-days SSS retrievals over the Pacific using an alternative Ocean Target Transformation applied to L1OP data," IFREMER/CLS, Tech. Rep., 2010.

[31] M. Martin-Neira, S. Ribo, and A. Martin-Polegre, "Polarimetric mode of MIRAS," Geoscience and Remote Sensing, IEEE Transactions on, vol. 40, no. 8, pp. 1755-1768, Aug 2002.

[32] A. Turiel, V. Nieves, E. García-Ladona, J. Font, M.-H. Rio, and G. Larnicol, "The multifractal structure of satellite temperature images can be used to obtain global maps of ocean currents," Ocean Science, vol. 5, pp. 447-460, 2009. 


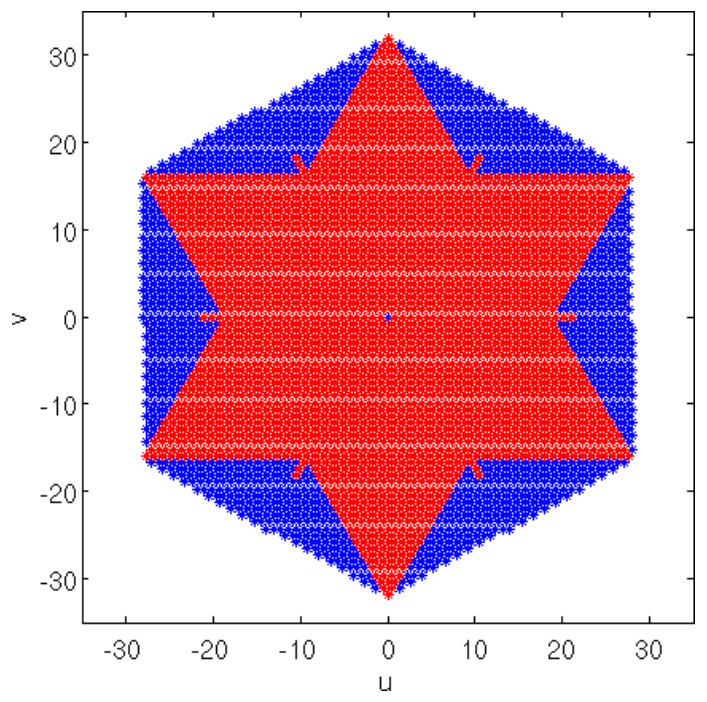

Fig. 1. Spatial frequencies where the visibility samples are measured (red stars). Zero-padding of the lacking spatial frequencies in the fundamental hexagon is performed before brightness temperature reconstruction (blue stars).

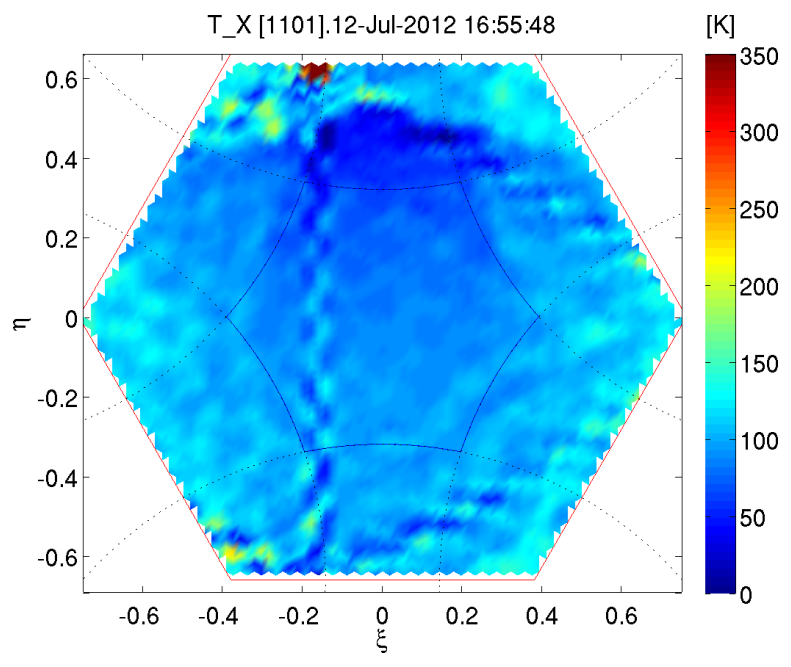

Fig. 2. Brightness temperature image over Pacific Ocean strongly affected by Sun aliases and tails. Nominal processing (G-matrix + Blackman window) has been used in the image reconstruction. 


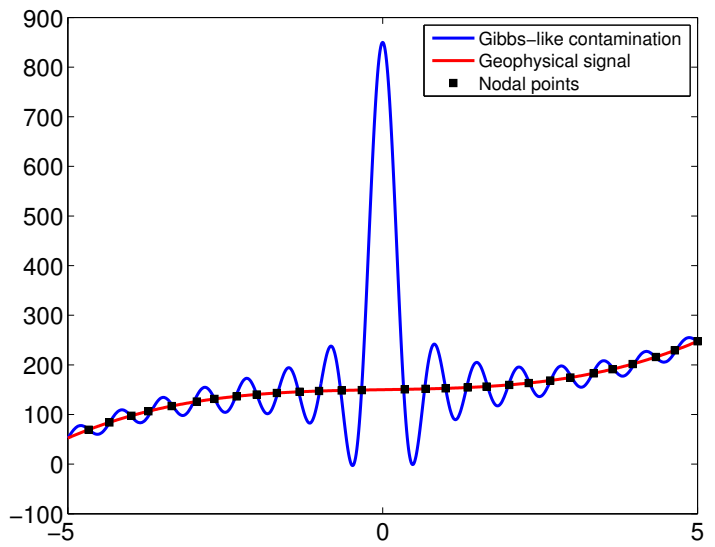

Fig. 3. 1D representation of the signal to be retrieved: a mixture of sharp transitions (Gibbs-like contamination), and the geophysical contribution (low contribution at high frequencies).

$[\mathrm{K}]$

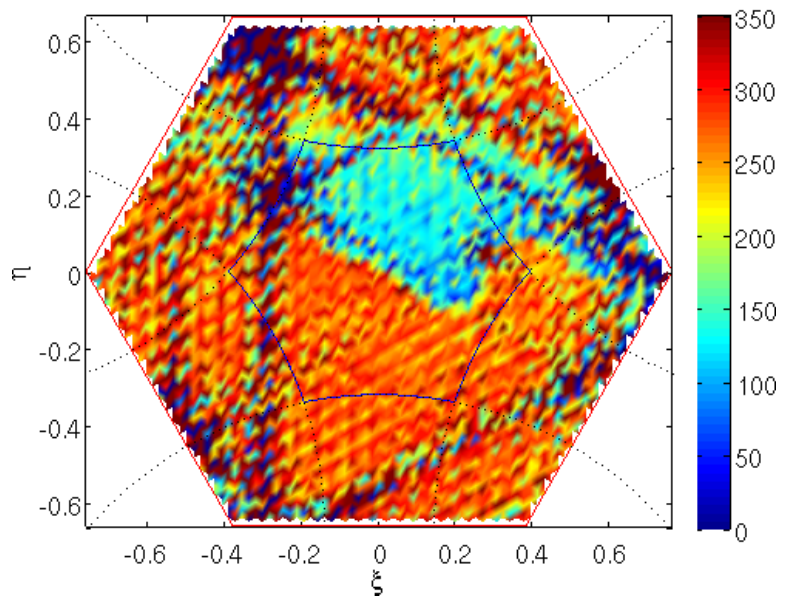

(a) SMOS reconstructed image (without Blackman window)

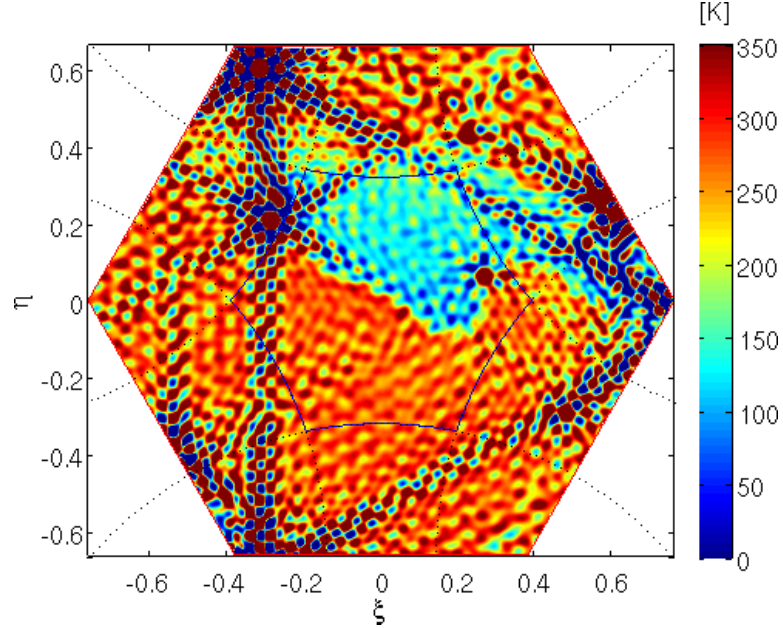

(b) Oversampled brightness temperature image

Fig. 4. Brightness temperature in the fundamental hexagon of a snapshot over North-Africa. Oscillating structures are more evident in the oversampled image. An oversampling factor $\beta=9$ has been used. 


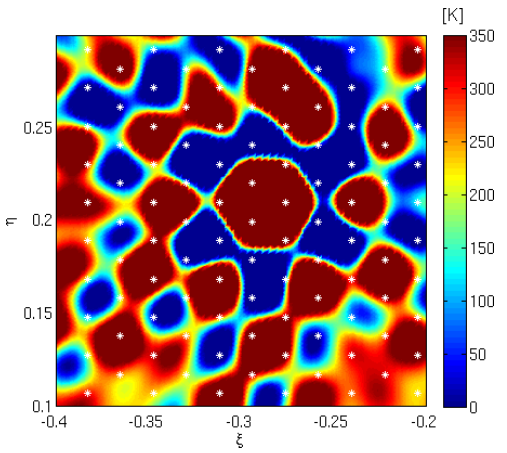

(a) SMOS original sampling

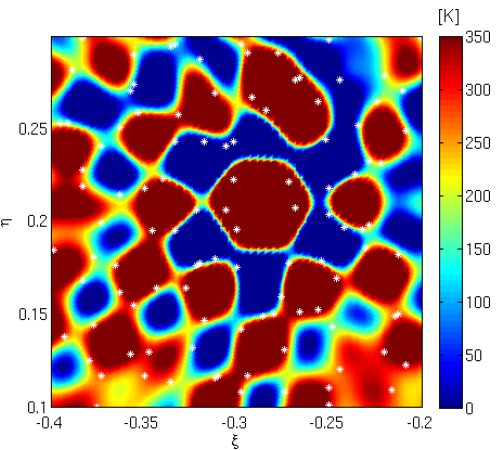

(b) First guess of nodal points

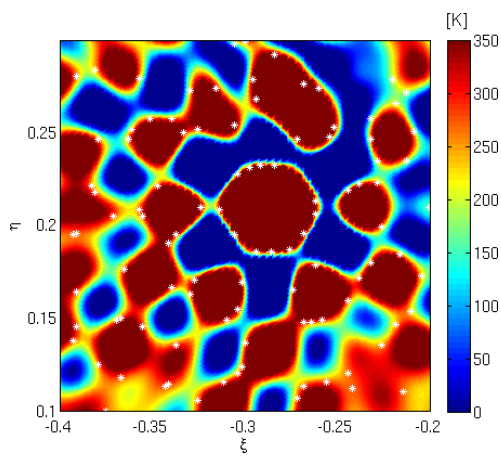

(c) Nodal points after 20 iterations

Fig. 5. Zoom of the oversampled brightness temperature image (without Blackman window) in Fig. 4b. White stars indicate the selected nodal points, that is, those points where the original image is sampled.

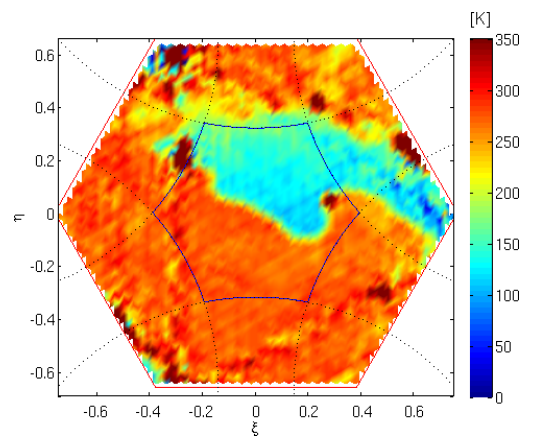

(a) Nominal TB

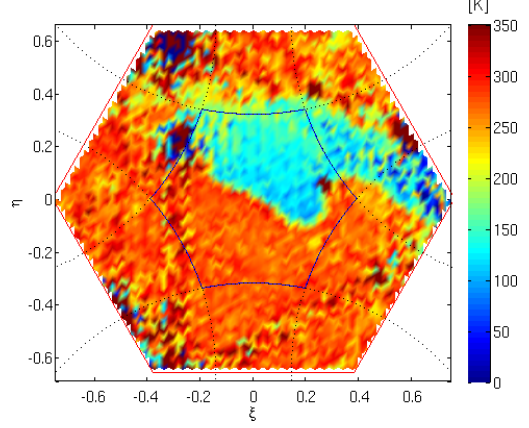

(b) NS TB, first guess

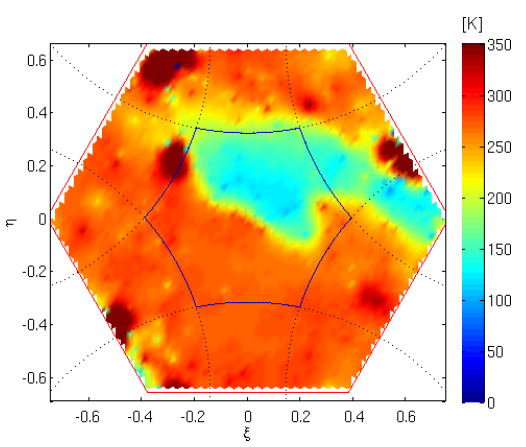

(c) NS TB, 20 iterations

Fig. 6. (a) Nominal brightness temperature image from Fig. 4. (b) Retrieved brightness temperature after applying nodal sampling technique, using subgrid in Fig. 5b. (c) Retrieved brightness temperature after applying nodal sampling technique, using subgrid in Fig. 5 c. An oversampling factor $\beta=9$ has been used in images (b) and (c). 


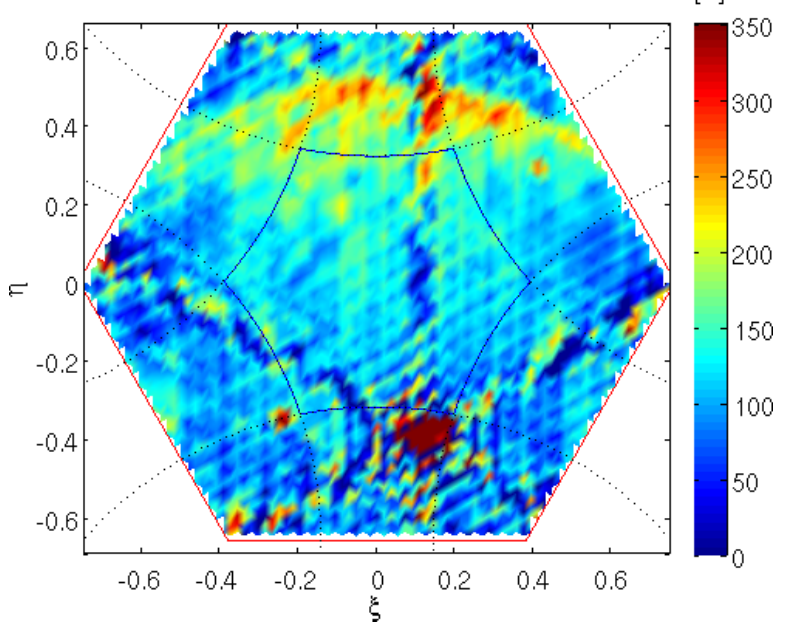

(a) Nominal TB

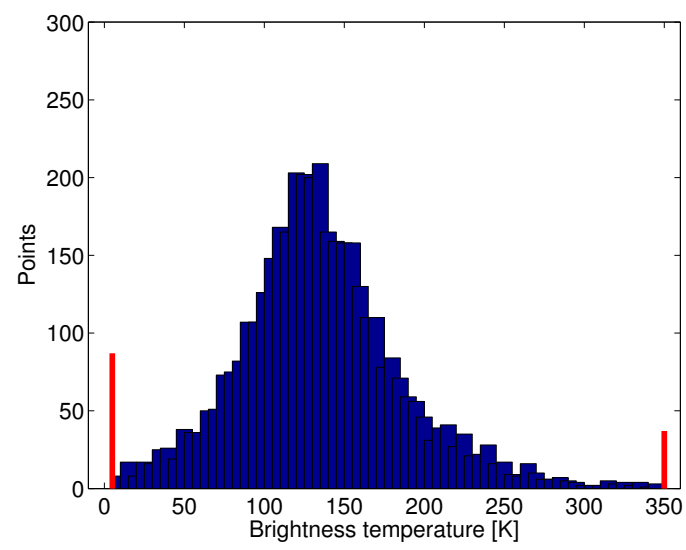

(c) Nominal TB histogram

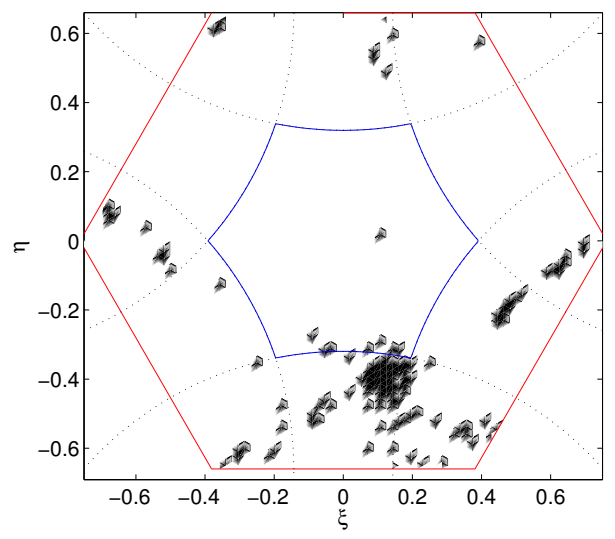

(e) Nominal TB mask

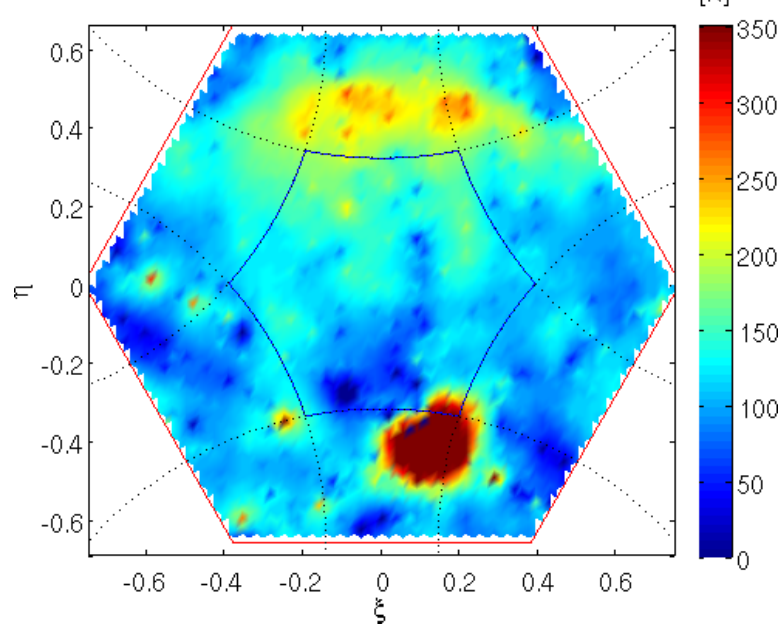

(b) Nodal sampling TB

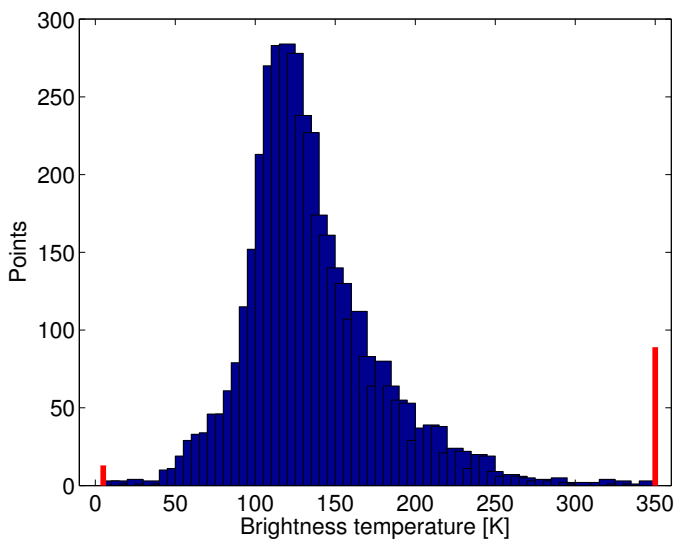

(d) Nodal sampling TB histogram

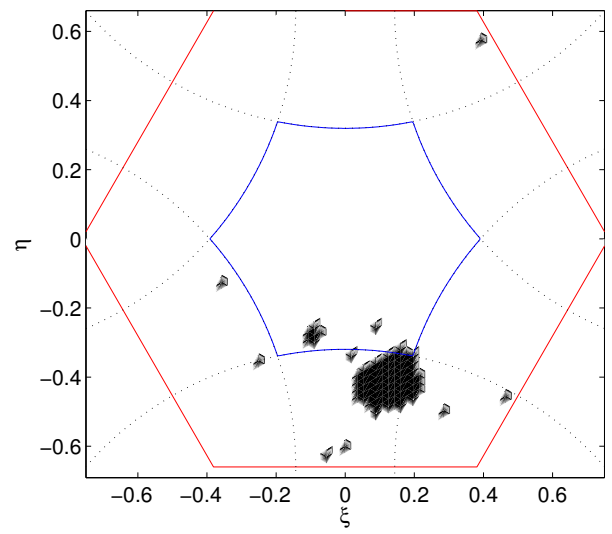

(f) Nodal sampling TB mask

Fig. 7. Brightness temperatures in the fundamental hexagon of a snapshot over the Atlantic Ocean strongly affected by an RFI produced by a ship, UTC time: 11-Feb-2010 21:46:25, Y-polarization. Red bars in the histograms account for negative brightness temperatures and values higher than $350 \mathrm{~K}$ (corresponding to non-natural emission). Pixels with TB values not corresponding to natural emission have been masked. 


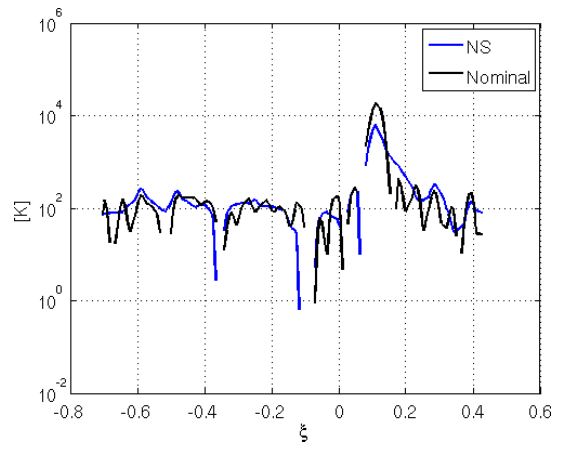

(a) Cut at $30 \mathrm{deg}$.

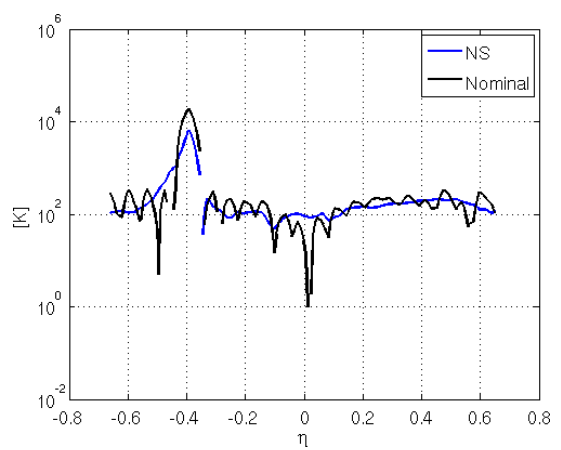

(c) Cut along eta

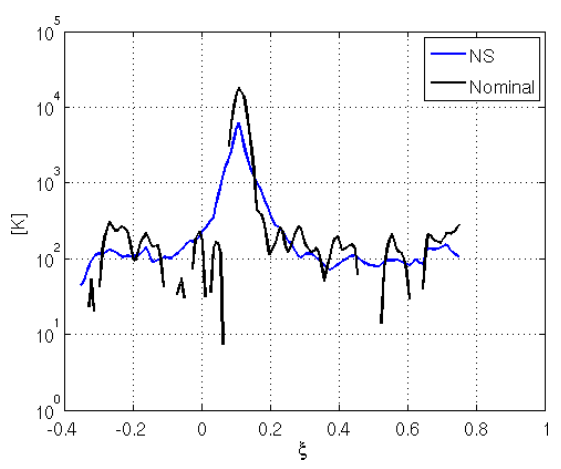

(b) Cut at $-30 \mathrm{deg}$.

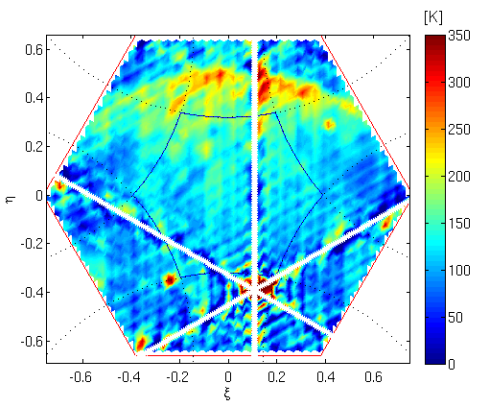

(d) Directions for TB cuts

Fig. 8. Cuts along the RFI directions of the brightness temperature image in Fig. 7. Note that the scale is logaritmic to show the full variability of brightness temperatures in this image contaminated by a strong RFI. A considerable decrease of the sidelobe levels can be appreciated when applying nodal sampling technique (blue line) with respect to the nominal one (black line).

$[\mathrm{K}]$

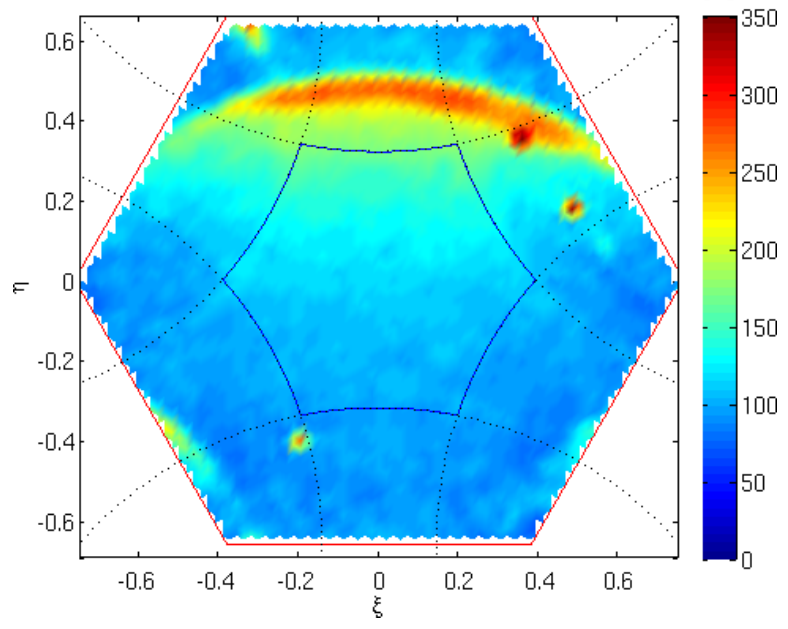

(a) Nominal TB
$[\mathrm{K}]$

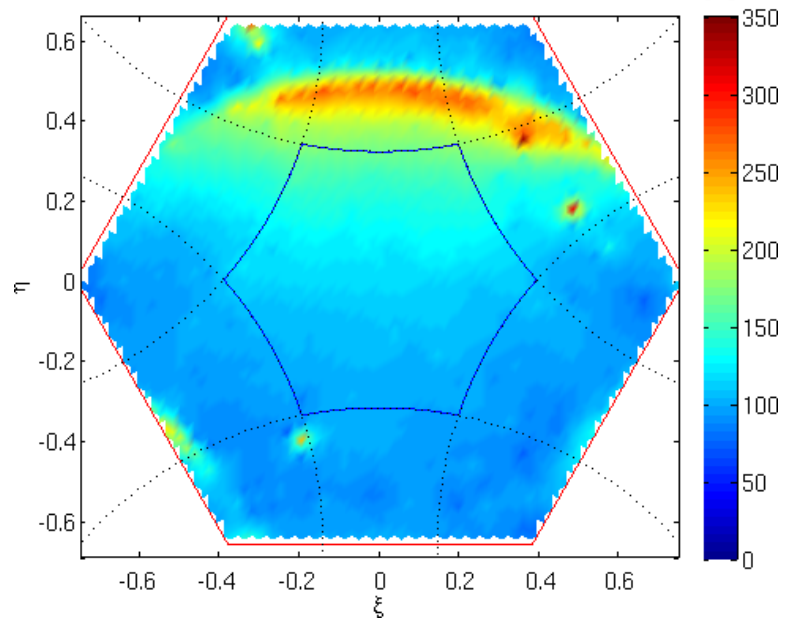

(b) Nodal sampling TB

Fig. 9. (a) Nominal brightness temperature image over the same zone than Fig. 7. (b) Retrieved brightness temperature after applying nodal sampling technique, using an oversampling factor $\beta=9$ and 20 iterations. 


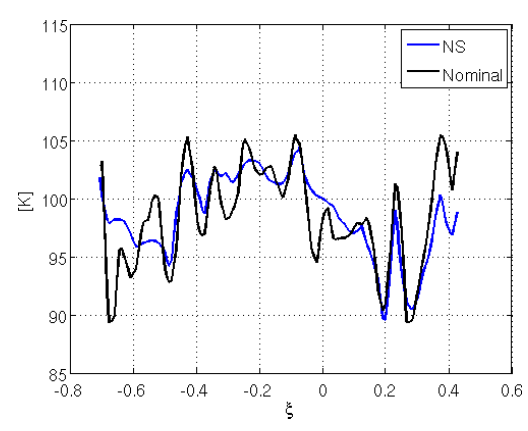

(a) Clean image, cut at $30 \mathrm{deg}$.

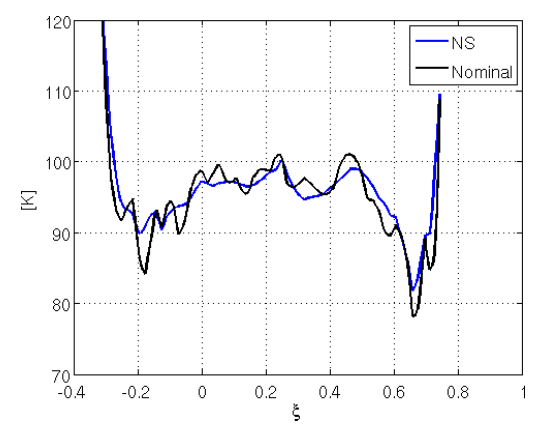

(b) Clean image, cut at $-30 \mathrm{deg}$.

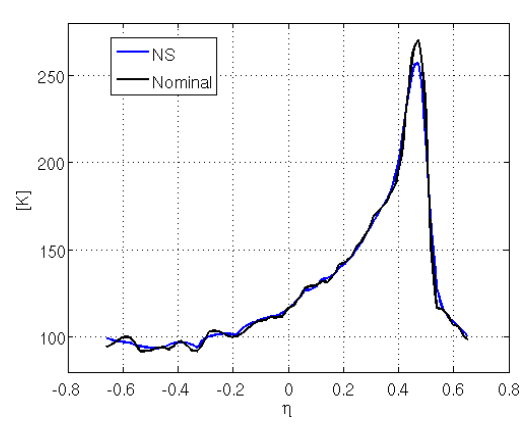

(c) Clean image, cut along eta

Fig. 10. Cuts of brightness temperature of the clean image in Fig. 9. Cuts of the brightness temperatures have been performed along the same directions shown in Fig. 8d. 


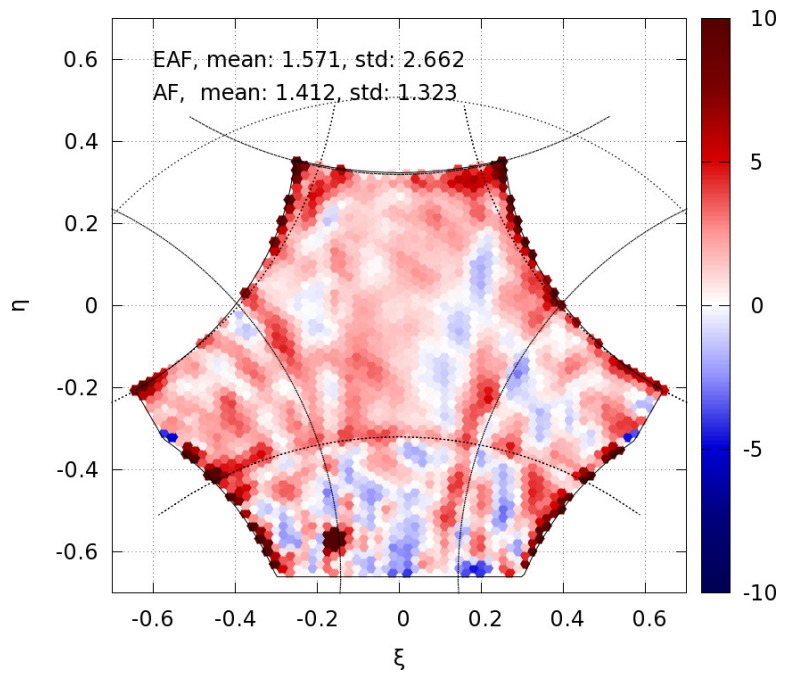

(a) Nominal, X-polarization

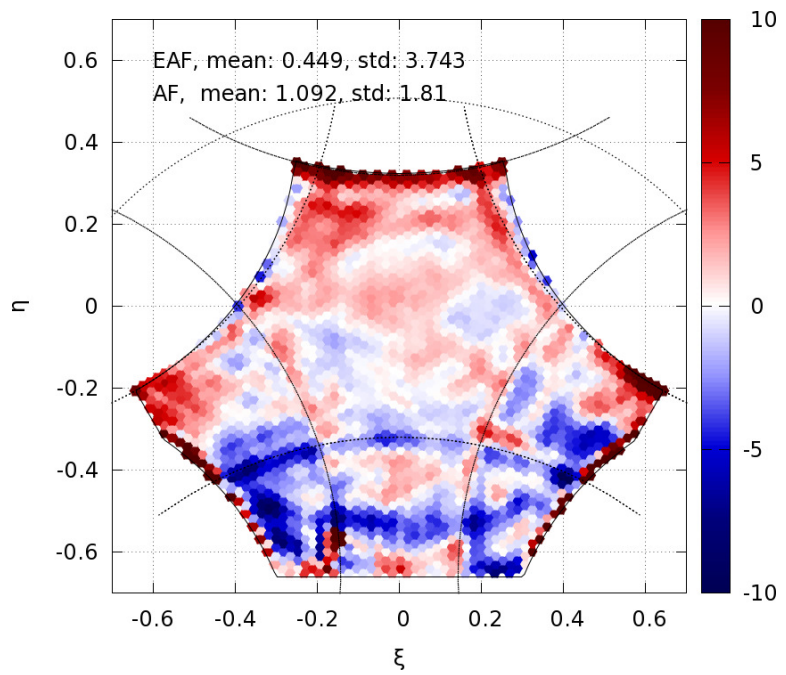

(c) Nominal, Y-polarization

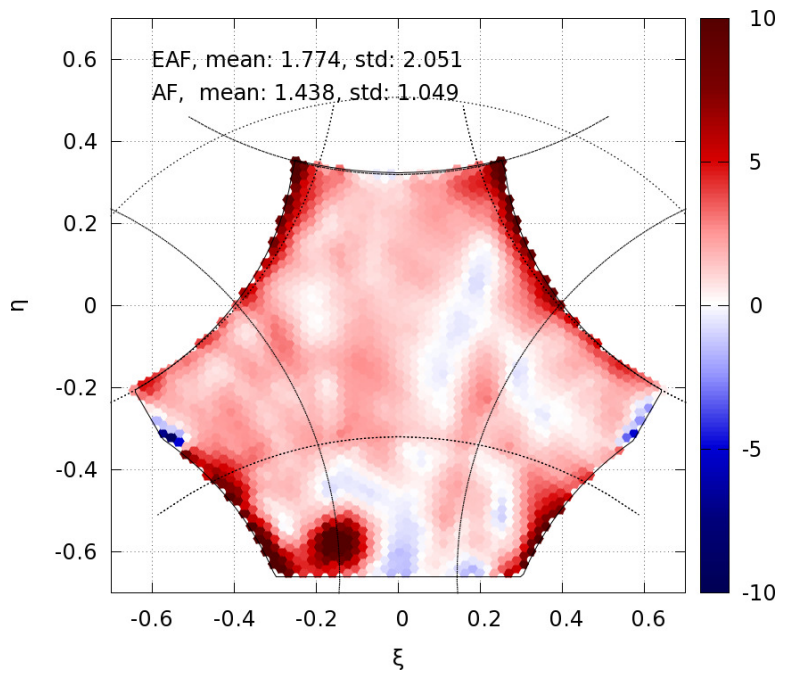

(b) Nodal sampling, X-polarization

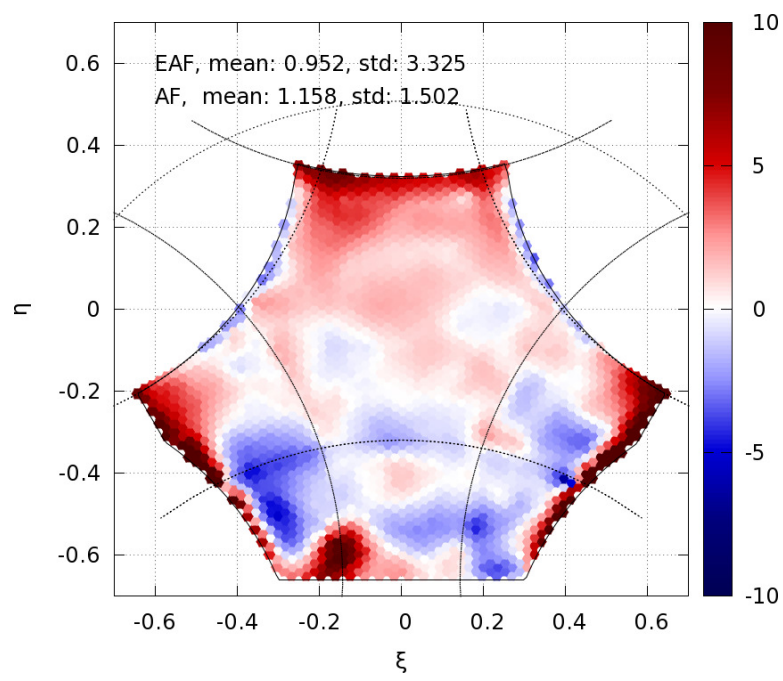

(d) Nodal sampling, Y-polarization

Fig. 11. Median of the difference between TB measurements and the theoretically modeled TB for the Extended Alias Free-Field of View (EAF-FOV). Statistics have been computed using an ascending orbit over a very stable zone in the Pacific Ocean (OTT computation zone). Dashed lines correspond to the the six unit circles aliases. 


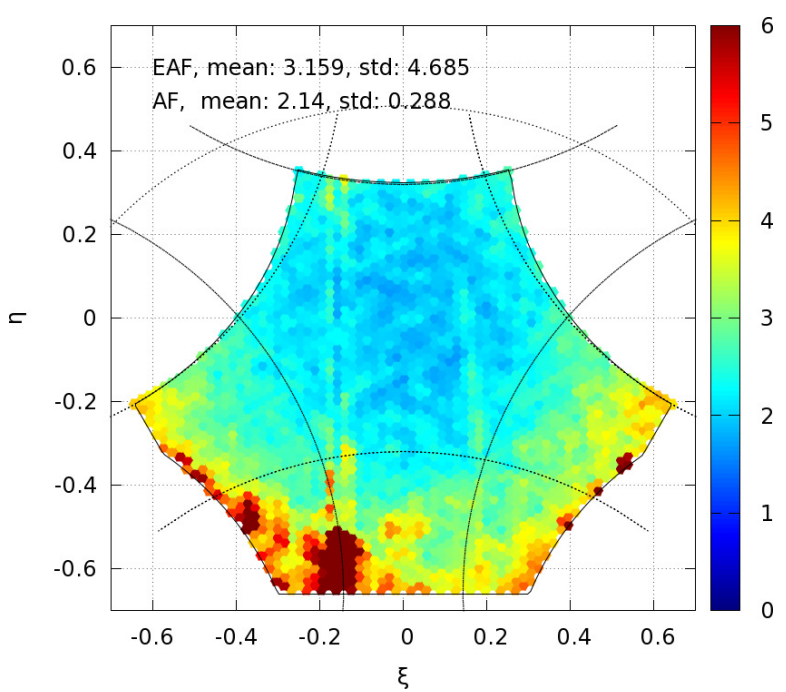

(a) Nominal, X-polarizattion

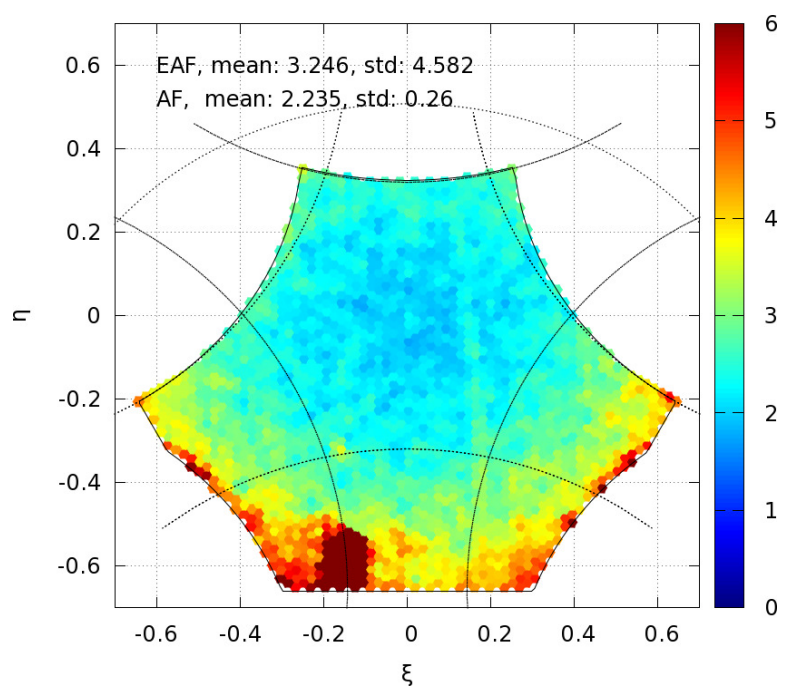

(c) Nominal, Y-polarization

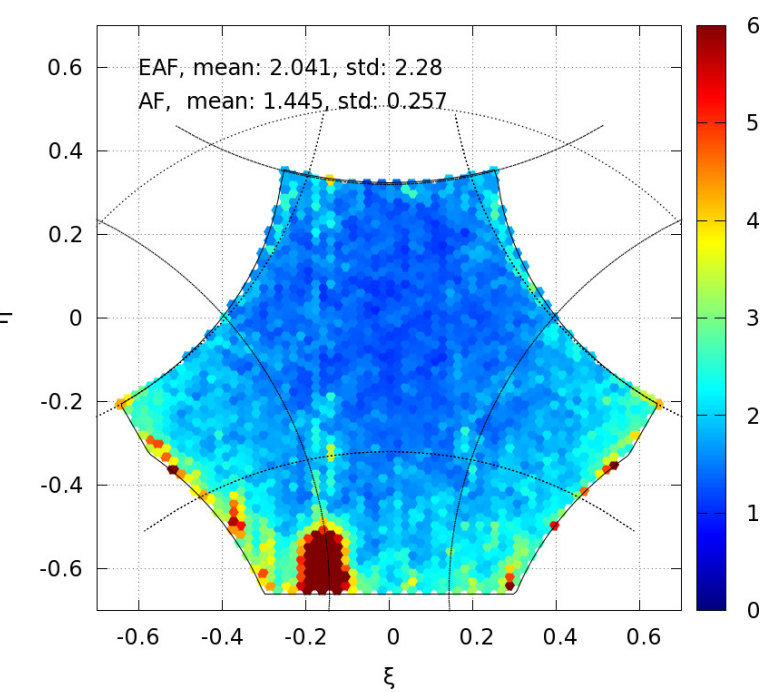

(b) Nodal sampling, X-polarization

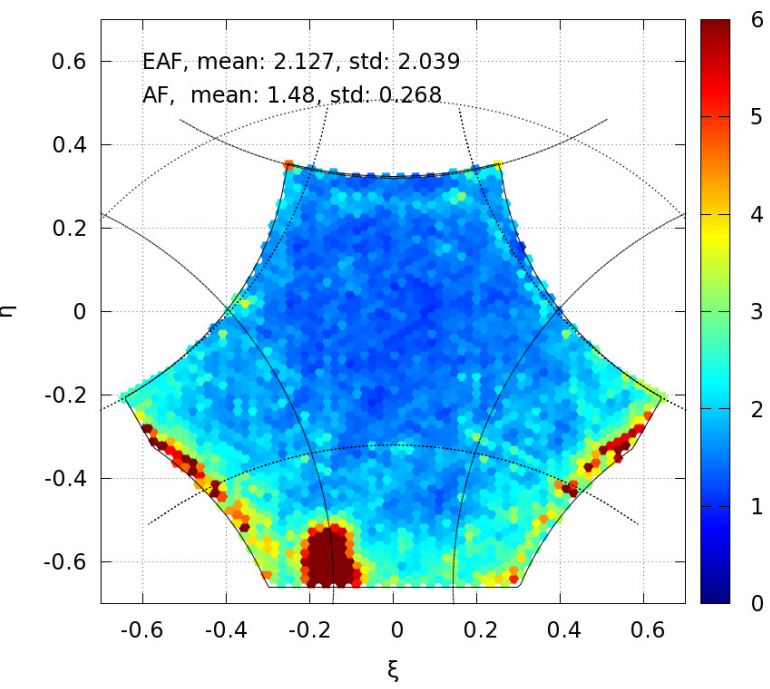

(d) Nodal sampling, Y-polarization

Fig. 12. Standard deviation of the difference between TB measurements and the theoretically modeled TB for the Extended Alias Free-Field of View (EAF-FOV). Statistics have been computed using an ascending orbit over a very stable zone in the Pacific Ocean (OTT computation zone). Sun alias and its tails can be clearly distinguished. Dashed lines correspond to the the six unit circles aliases. 


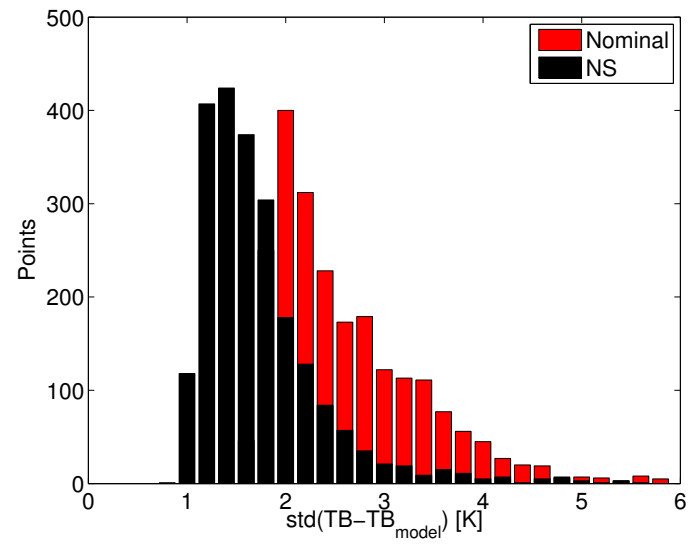

(a) X-polarizattion

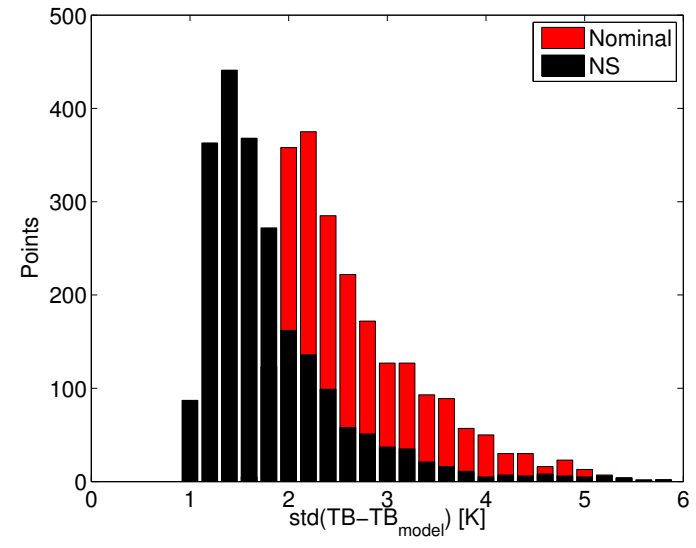

(b) Y-polarization

Fig. 13. Distributions of the standard deviation of the difference between the TB measurements and the model for nominal (red) and nodal sampling (black) processing. A reduction of approximately $1 \mathrm{~K}$ is obtained when using nodal sampling technique with respect to the SMOS nominal processing.

First Stokes: TB SMOS - TB model

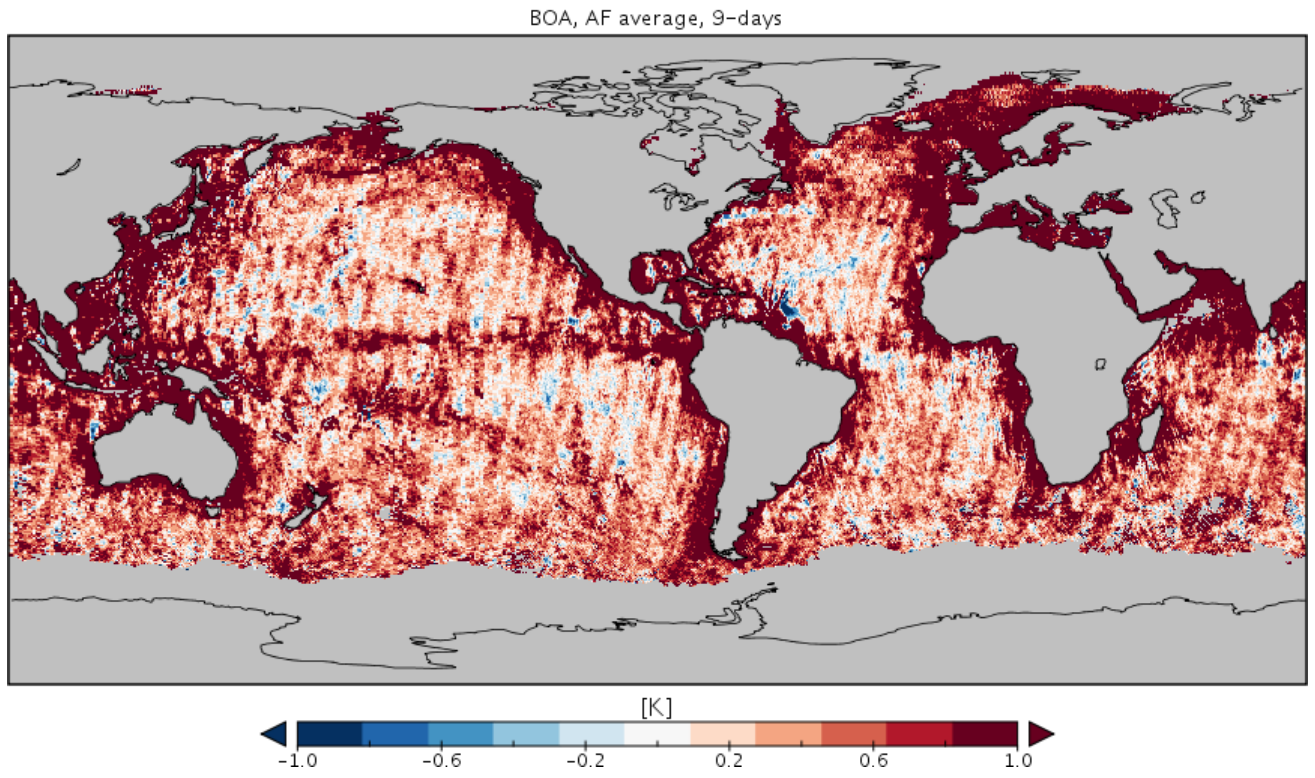

Fig. 14. 9-day $0.25^{\circ}$ resolution map of the First Stokes brightness temperatures difference between SMOS measurements and the model for nominal image reconstruction. Brightness temperatures at BOA have been used to produce the map. The average for all the pixels in the AF-FOV region has been performed. 
First Stokes: TB SMOS - TB model

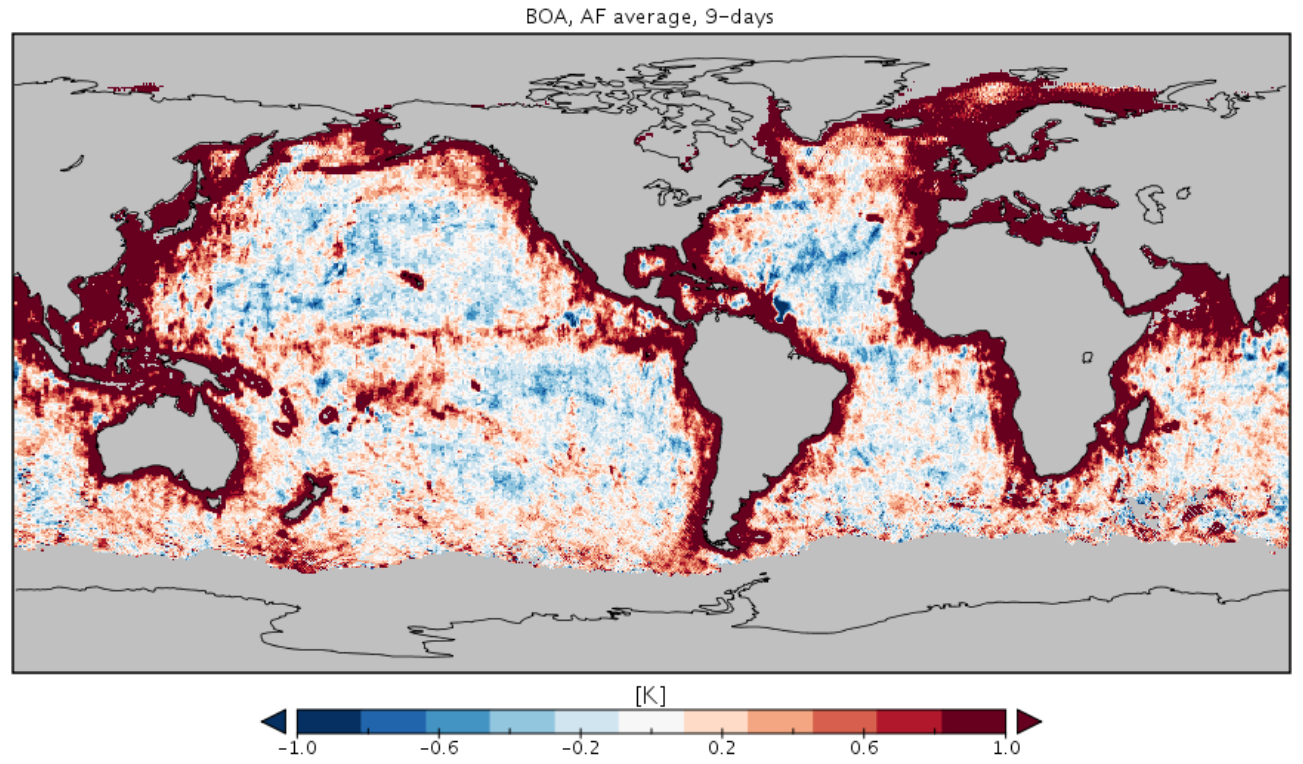

Fig. 15. 9-day $0.25^{\circ}$ resolution map of the First Stokes brightness temperatures difference between SMOS measurements and the model for nodal sampling approach. Brightness temperatures at BOA have been used to produce the map. The average for all the pixels in the AF-FOV region has been performed.

Standard deviation of First Stokes difference: TB SMOS - TB model

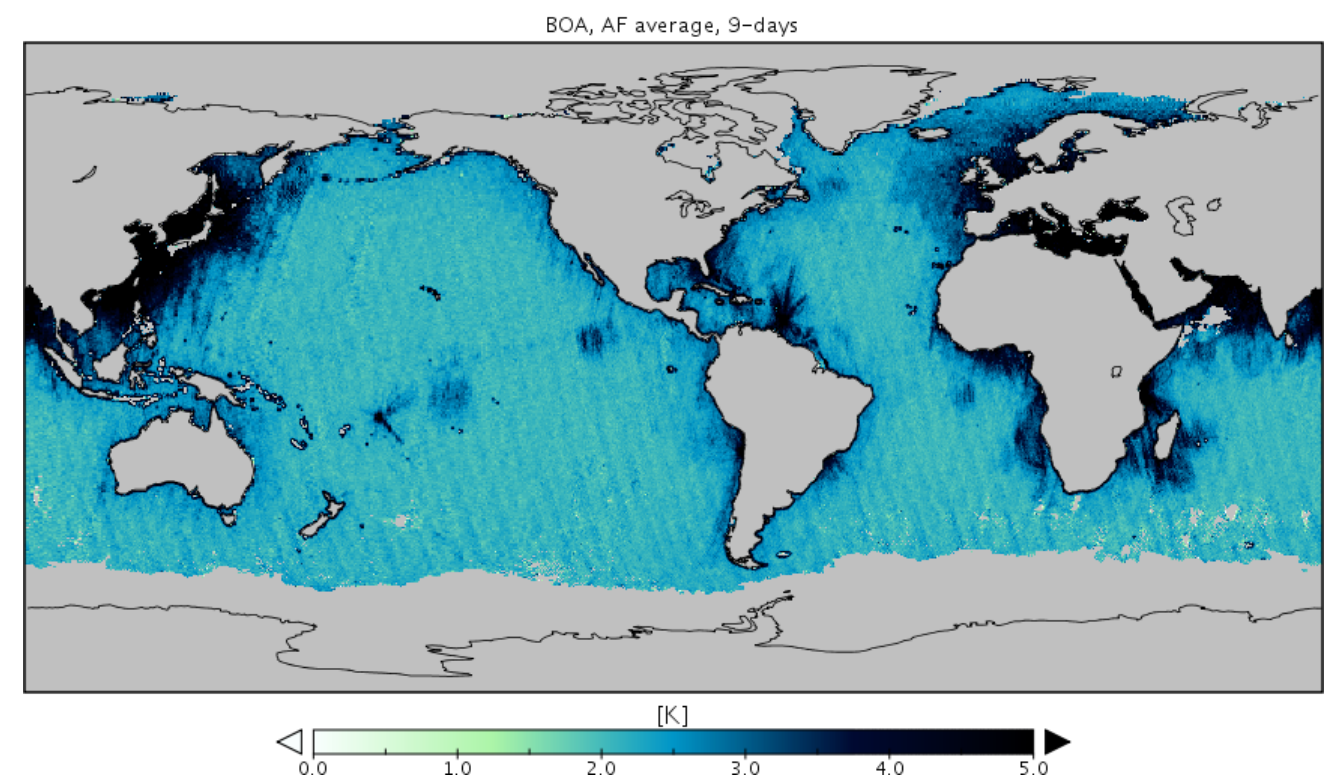

Fig. 16. 9-day $0.25^{\circ}$ resolution map of the standard deviation of the First Stokes brightness temperatures difference (SMOS measurements minus model) for nominal image reconstruction. Brightness temperatures at BOA have been used to produce the map. The average for all the pixels in the AF-FOV region has been performed. 
Standard deviation of First Stokes difference: TB SMOS - TB model

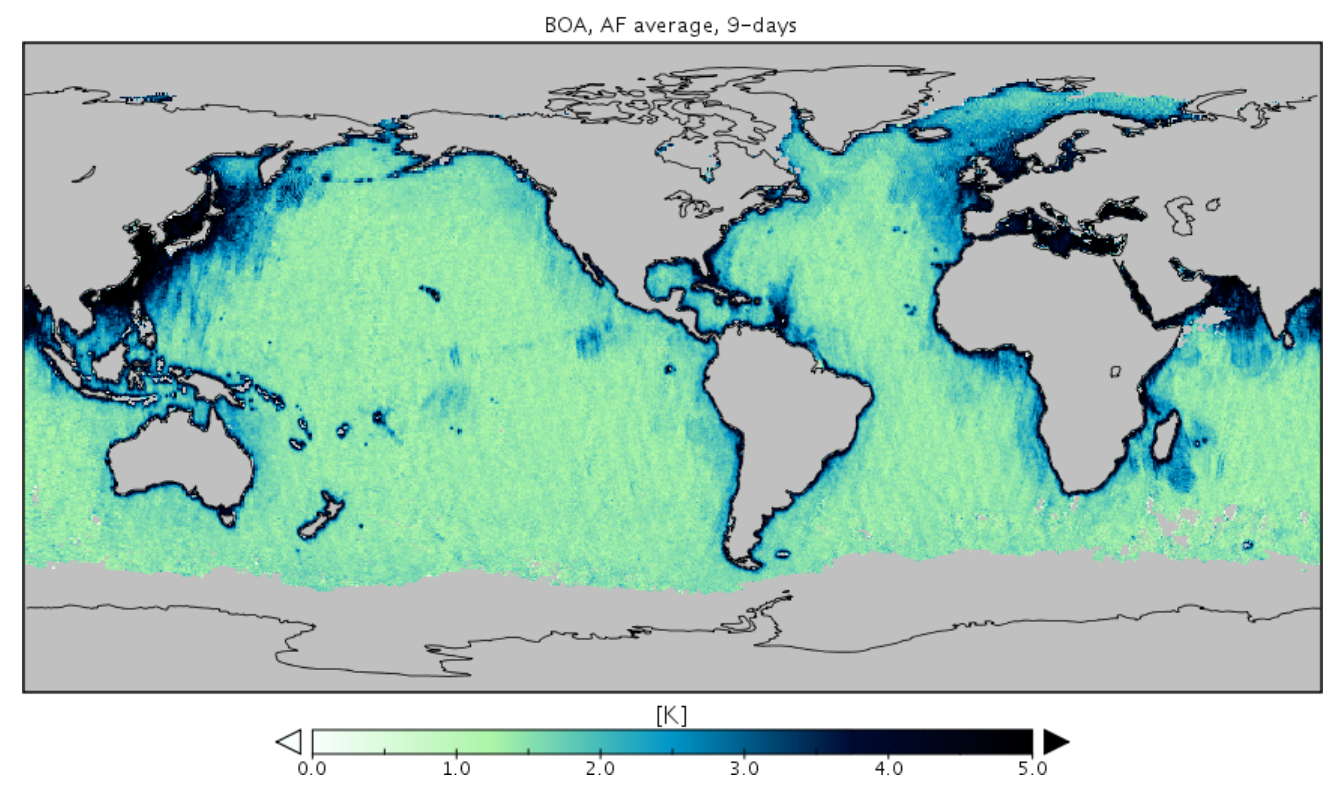

Fig. 17. 9-day $0.25^{\circ}$ resolution map of the standard deviation of the First Stokes brightness temperatures difference (SMOS measurements minus model) for nodal sampling approach. Brightness temperatures at BOA have been used to produce the map. The average for all the pixels in the AFFOV region has been performed. 